UPPSALA UNIVERSITET
Working Paper 2009:8

Department of Economics

Nonparametric Structural Estimation of Labor Supply in the Presence of Censoring

Che-Yuan Liang 
Department of Economics

Working paper 2009:8

Uppsala University

May 2009

P.O. Box 513

ISSN 1653-6975

SE-751 20 Uppsala

Sweden

Fax: $+{ }_{4} 6184711478$

Nonparametric Structural Estimation of

Labor Supply in the Presence of Censoring

Che-YuAn LiAng

Papers in the Working Paper Series are published on internet in PDF formats.

Download from http://www.nek.uu.se or from S-WoPEC http://swopec.hhs.se/uunewp/ 


\title{
Nonparametric Structural Estimation of Labor Supply in the Presence of Censoring*
}

\author{
Che-Yuan Liang ${ }^{\#}$ \\ May 18, 2009
}

\begin{abstract}
This paper extends the nonparametric method to estimate labor supply developed by Blomquist and Newey (2002) to handle cases in which there are individuals who do not work. The method is then applied to married women in Sweden from 1973 to 1999. For 1999, I find an aggregate uncompensated wage elasticity around 1 and an aggregate income elasticity around -0.05 . Furthermore, marginal tax rates are beyond the net government revenue maximizing rates. Despite large labor supply effects, the dramatic evolution of the tax system can only explain a small share of the 58 percent rise in female labor supply during this period.
\end{abstract}

Keywords: female labor supply, nonparametric estimation, nonlinear budget sets, tax revenues

JEL classification: C14, D31, H31, J22

\footnotetext{
${ }^{*}$ I thank Spencer Bastani, Sören Blomquist, Mikael Elinder, Erik Glans, Per Johansson, Henrik Jordahl, Mikael Lindahl, Whitney Newey, Panu Poutvaara, Håkan Selin, and seminar participants at Uppsala University, Stockholm University, and Research Institute of Industrial Economics for valuable comments and suggestions, Martin Hällsten and Michael Tåhlin for preparing and providing me some of the data used, Sören Blomquist and Matias Eklöf for providing me descriptions and codes over tax systems, as well as the Jan Wallander and Tom Hedelius Foundation and the Uppsala Center for Fiscal Studies for financial support.

\# Uppsala Center for Fiscal Studies, Department of Economics, Uppsala University, P.O. Box 513, SE-75120 Uppsala, Sweden; Phone: +46-(0)18-4711633; Fax: +46-(0)18-4711478; E-mail: che-yuan.liang@nek.uu.se.
} 


\section{Introduction}

The responsiveness of labor supply to taxation is of great interest to policymakers, as it is crucial for the welfare evaluation of tax systems. There are many difficulties involved in estimating labor supply, and there exists a large literature that uses different approaches with varying results. Today, there is a general belief that male labor supply is not very responsive, but there is no consensus regarding female labor supply. Blundell and MaCurdy's (1999) survey of the literature finds uncompensated wage elasticities ranging from 0 to 2 and income elasticities ranging from -1 to 0.5 for women. The bounds are so widespread that they are useless for policy evaluation. This lack of convergence and understanding of the reasons for the dispersion illustrates the need for additional methodological developments in this area of research.

This paper contributes by extending the nonparametric method developed by Blomquist and Newey (2002) to handle censoring, which allows estimation for groups with considerable numbers of individuals who choose the corner solution of zero hours of work. The method is then applied to married women in Sweden from 1973 to 1999.

Compared to other countries, Sweden's tax burden is and has generally been very high. Its total tax revenues as share of gross national product have been consistently among the top two (with Denmark) among the OECD countries during the sample period (OECD, 2008). A testable hypothesis that is relevant to countries with a high tax burden and to groups with high labor supply responsiveness is whether tax rates are beyond the net government revenue maximizing rates. This would be a nearly indisputable sign of suboptimal tax rates as rate reductions would lead to government revenue gains. Most studies from the 1980s produced labor supply elasticities that would reject this hypothesis for prime-aged working men (see, e.g., MaCurdy, 1992). More recent studies that focus on taxable income find more support, especially for men with high incomes (see, e.g., Goolsbee, 1999; and Mofitt and Wilhelm, 2000). For female labor supply, there is much less consensus. The empirical application in this paper provides an assessment on this issue for a large and economically important group, which is considered to be more sensitive to taxation than married men and faces very high tax rates.

There have been several sweeping reforms to the Swedish tax system during the period of study. The overall tax level increased in the 1970s to a historical peak in 1980, decreased during the 1980s to a historical low in 1991, and finally increased a little since then. The tax system did not change much in the 2000s until the earned income tax credit reform in 2007. 
During this period, the labor supply of married women also rose from an average level of 55 percent to 87 percent of regular full-time hours (a 58 percent increase), which nearly bridges the entire gap between them and their almost exclusively full-time working husbands. The empirical application in this paper also provides an assessment of the role of tax policy in raising female labor supply and in creating gender equality at work. This is of great policy interest, as the labor markets in many OECD countries today are similar to that of 1973 Sweden (OECD, 2009).

Like the main strand in the literature, I depart from a static secondary-earner framework; i.e., I do not account for dynamic savings concerns and I account for the income of the other spouse as if it were unearned income. There are, even within this framework, several major difficulties in estimating the effects of taxation on labor supply. One is that there are selection problems in gross wages and unearned incomes as they typically covary with observable or unobservable variables that may have their own effects on the labor supply. Another difficulty arises because the nonlinear tax and transfer system, with its many tax brackets, creates nonlinear budget sets that have many segments. Individuals can therefore affect the marginal tax rate that they face by selecting which segment to place themselves in. Hours of work and the net tax are therefore simultaneously determined. The nonlinearities also create kinks in the budget set around which there is no net wage. An additional difficulty is that the functional form of the labor supply is unknown. Furthermore, some methods depend on distributional assumptions that may drive the estimation results. In the case of female labor supply, there is also substantial censoring.

The most straightforward strategy to estimate labor supply is to linearize the budget set around the actual marginal segment where individuals place themselves and to estimate the effects of this segment on the labor supply with ordinary least squares (OLS). This approach suffers from most of the mentioned difficulties.

Approaches that primarily focus on credible identification often solve the selection issue convincingly. The natural experiment approach uses within-group or within-individual variations provided by tax reforms that hit groups or individuals differently (e.g., Eissa, 1995; and Feldstein, 1995). Estimation is carried out with difference-in-differences or first-difference methods. Unfortunately, this approach leaves the nonlinear budget set issues unsolved. Additional functional form assumptions are also needed to obtain general behavioral elasticities.

The instrumental variables approach could additionally handle the simultaneity issue (see, e.g., Pencavel 1986), although it leaves the functional form issue unsolved. Nonetheless, Mroz (1987) and Blomquist (1996) show that the results are very sensitive to the set of in- 
struments used, which indicates that the exclusion restriction and instrument strength assumptions are seldom fulfilled at the same time. Several recent studies combine natural experiments and instrumental variables (e.g., Auten and Caroll, 1999; and Gruber and Saez, 2002). However, Mofitt and Wilhelm (2000) point out that it seems unlikely that the simultaneity issue is completely solved in those studies.

Structural approaches that focus on tight connection between empirical specification and theory often solve the nonlinear budget set issues convincingly and are also useful for simulations at the expense of selection problems. The maximum-likelihood (ML) based approach developed in Burtless and Hausman (1978), Hausman (1981), and Hausman (1985), henceforth referred to as the Hausman approach, was very popular for a long time. Nonlinearities are accounted for by entering variables characterizing the whole budget set into the likelihood function. Desired hours of work are usually assumed to be the outcome of utility maximization, subject to piecewise-linear convex budget constraints, to make computations feasible and interpretation straightforward. The standard method assumes joint normality of two error terms. However, Blomquist and Newey (2002) report evidence that these distributional assumptions may not hold and that that the bias could be quite large (67 percent in their application). Additionally, the choice of functional form produces nontrivial differences (see, e.g., Blomquist and Hansson-Brusewitz, 1990).

Another increasingly popular ML-based approach is to treat the hours of work decision as a discrete choice problem and to use multiple choice methods (e.g., van Soest, 1995; and Hoynes, 1996). A potential pitfall is that measurement errors in hours of work cannot be accommodated. This approach also requires distributional assumptions, and there is some evidence that limited dependent variable models in general are sometimes sensitive to them (see, e.g., Chay and Powell, 2001; and Wooldridge, 2002). Functional form assumptions are also needed, although these could be quite flexible (e.g., van Soest et al., 2002).

Blomquist and Newey (2002) recently developed a nonparametric structural approach that handles all the mentioned difficulties except the selection issue when there is no censoring. The whole budget set is accounted for without imposing a functional form on the labor supply and without requiring distributional assumptions. Many variables are required to characterize the whole budget set, resulting in a "curse of dimensionality" that needs to be circumvented for nonparametric estimation to be feasible. They overcome this problem by putting structure on the regression with utility maximization restrictions in piecewise-linear convex budget sets. Estimation is carried out with OLS. Blomquist et al. (2001), Wu (2005), and Kumar $(2006,2007)$ are the only others who have applied this approach. 
The first part of this paper extends their nonparametric method to the case with censoring. In addition to estimation of expected hours of work, the extension enables estimation of the participation probability within the same framework, which makes it possible to sort out responses on the participation and hour margins. This paper is closely related to that by Kumar (2006), who also attempts to develop such an extension. For expected hours of work, I arrive at a rather different labor supply function, although the empirical specifications that we use are both similar to the original specification by Blomquist and Newey (2002). It is my opinion that, although he accounts for censoring in desired hours of work, it is more appropriate to account for censoring in observed hours of work, which include measurement errors. To sort out responses on the participation and hour margins, I derive and estimate a structural participation function within the original framework, whereas he applies the nonparametric sample correction method suggested by Das et al. (2003).

The second part of this paper then uses the proposed extension to estimate the labor supply of married or cohabiting women (henceforth referred to as married women) in Sweden from 1973 to 1999 with data from four years. My empirical specification is an improvement over Blomquist and Newey's (2002) in that I add demographic control variables and time dummies. By primarily relying on the variation in impact of tax reforms on individual budget sets for identification, like in the natural experiment approach, selection issues are dealt with more carefully than previous structural studies. The controls are included nonparametrically and structurally unlike in the work of $\operatorname{Kumar}(2006,2007)$ on American data. This flexibility allows the responsiveness not only to be a function of the tax system and the tax reform of interest but also of demographics and time factors. In contrast to the constant elasticity assumption often made in the literature, there is some evidence that labor supply elasticities vary with tax system and time (e.g., Goolsbee, 1999; and Heim, 2007).

Labor supply estimates are usually summarized in terms of elasticities for linear budget sets. At the sample mean, I find a statistically significant uncompensated wage elasticity of labor supply around 0.7 and a small income elasticity for linear budget sets. However, the wage elasticity varies between 0.3 and 0.8 depending on the point of evaluation. More problematic is that the budget sets are nonlinear. There are therefore many net wage rates, and it is usually not possible to vary one rate in isolation. Extrapolation is also needed to infer behavior on linear budget sets. It is more appropriate to use the labor supply estimates for simulations with realistic budget sets.

These problems lead me to construct simulation-based aggregate elasticity measures, specific to each tax system, population, and type of reform. Such measures are not as general 
as, nor directly comparable to, linear budget set elasticities, but they are much more informative and policy relevant. I simulate hypothetical reforms and construct such elasticities for the 1999 tax system, which is similar to the present Swedish system and almost identical to the 2007 system prior to the latest earned income tax credit reform. I find an aggregate uncompensated wage elasticity of 0.9 for marginal tax rate changes in low and medium income brackets with roughly equal contributions from the participation and hour margins; in high income brackets, my finding is 1.4 . The linear budget set wage elasticity at the mean is therefore an underestimation of the aggregate wage elasticity. The hour margin contributes as much as the participation margin at low and medium income brackets, but four times more at high income brackets. I also find an aggregate income elasticity of -0.05 with the bulk made up of responses on the hour margin. These findings suggest that the participation rate is very responsive both to the changes in the first marginal segment that non-working individuals face and to the rest of the budget set.

The simulations reveal negative average tax elasticity of net government revenues at all income levels: -0.3 in low and medium income brackets and -1.5 in high income brackets. This indicates that marginal tax rates are beyond the revenue maximum for married women.

In parts of the simulations, I also account for the mechanical effects of the hypothetical reforms on the husbands' incomes and the income cross-effects that these have. The small income elasticity that I find for married women and that Blomquist and Newey (2002) find for married men indicates that such effects are small for both spouses in Sweden. The mechanical effects on the husbands have, however, large financial effects on net government revenues. Accounting for these effects produces marginal tax rates that are on the right side of the revenue maximum at the household level. Additionally accounting for the behavioral effects of the husbands, using back-of-the-envelope calculations based on the labor supply estimates and simulation results for married Swedish men (Blomquist et al., 2001), gives marginal tax rates that are on the right side in the low and medium income brackets but at the revenue maximum in the high income brackets. A comparison of the spouses shows that the financial effects on the wives to which their behavioral effects translate are much larger than those on their husbands, despite the wives' lower gross wages.

Although there are large behavioral effects of taxation, simulations also reveal that the dramatic evolution of the tax system can only explain a small share of the extreme rise in female labor supply during the sample period. It cannot explain any of the rise prior to 1981 , but it can account for 34 percent of the rise afterwards. Demographics and time factors, such as 
attitudes, technology, and macroeconomics, account for the main part of the rise. Variation in gross incomes also contributes non-trivially.

The paper proceeds as follows. The next section derives the labor supply functions. Section three describes how the functions are estimated empirically. Section four describes the data. Section five reports the labor supply estimates and sorts out tax system effects during the sample period. Section six simulates hypothetical reforms and aggregate elasticities. The last section concludes.

\section{Labor Supply Functions}

Blomquist and Newey (2002) develop a method to nonparametrically estimate expected hours of work conditional on a nonlinear budget set, i.e., to estimate $E\left(h_{i} \mid \boldsymbol{x}_{i}\right)$, where $h_{i}$ is observed hours of work, $\boldsymbol{x}_{\boldsymbol{i}}$ is a vector of income variables characterizing the whole budget set, and subscript $i$ is an index for individuals. $E\left(h_{i} \mid \boldsymbol{x}_{i}\right)=s\left(\boldsymbol{x}_{i}\right)$ is well defined as it is, but nonparametric estimation is infeasible due to the high dimension of $\boldsymbol{x}_{\boldsymbol{i}}$. To overcome this problem, they reduce this function into sums of additive separable low-dimensional functions that are each feasible to estimate nonparametrically. This derivation is done under the assumption of utility maximization subject to piecewise-linear convex budget constraints.

A simplifying assumption that they make is that hours of work are unconstrained; i.e., there is no censoring. This is a good approximation for the labor supply of married men that they estimate, but not for, e.g., married women. The participation rate is 90.7 for married women in the sample used here, whereas it is 99.1 for a similar sample of married men. I derive a similar expression when some individuals do not work, i.e., when there is censoring at zero hours of work. I also derive the participation probability that could be used to estimate the responsiveness on the participation margin.

I follow their approach closely, but I also condition on a vector of control variables $\boldsymbol{z}_{i}$, and therefore derive $E\left(h_{i} \mid \boldsymbol{x}_{i}, z_{i}\right)$ and $\operatorname{Pr}\left(h_{i}>0 \mid \boldsymbol{x}_{i}, z_{i}\right)$. Adding control variables increases the dimensionality. Blomquist and Newey (2002) argue that parameters of some demographic variables are insignificant for married men when using a parametric approach, and they do not use any control variables. However, it is generally believed that demographics play a larger role for the labor supply of married women, particularly for the participation decision. There may also be strong effects of attitudes, technology, and macroeconomics on the labor supply.

Expected hours of work conditional on working can then be recovered as 


$$
E\left(h_{i} \mid \boldsymbol{x}_{\boldsymbol{i}}, \boldsymbol{z}_{i}, h_{i}>0\right)=\frac{E\left(h_{i} \mid \boldsymbol{x}_{\boldsymbol{i}}, \boldsymbol{z}_{\boldsymbol{i}}\right)}{\operatorname{Pr}\left(h_{i}>0 \mid \boldsymbol{x}_{\boldsymbol{i}}, \boldsymbol{z}_{\boldsymbol{i}}\right)}
$$

and can be used to examine the responsiveness on the hour margin. In elasticity terms, $E l_{E(h)}=$ $E l_{\operatorname{Pr}(h>0)}+E l_{E(h \mid h>0)}$, where $E l$ denotes own elasticities, which can be derived from equation (1) and the decomposition in McDonald and Mofitt (1980). The reason for not deriving $E\left(h_{i} \mid \boldsymbol{x}_{\boldsymbol{i}}, \boldsymbol{z}_{i}, h_{i}>0\right)$ and retrieving one of the other two entities using equation (1) is that this derivation results in an expression that is too complicated to estimate nonparametrically.

The labor supply function takes the form

$$
h_{i}^{*}=h^{*}\left(\boldsymbol{x}_{\boldsymbol{i}}, \boldsymbol{z}_{\boldsymbol{i}}, v_{i}, \varepsilon_{i}\right)=\pi_{i}^{*}+\varepsilon_{i}=\pi^{*}\left(\boldsymbol{x}_{\boldsymbol{i}}, \boldsymbol{z}_{\boldsymbol{i}}, v_{i}\right)+\varepsilon_{i} .
$$

$v_{i}$ is unobserved heterogeneity and captures the remaining variation in utility functions across individuals once $z_{i}$ has been accounted for. Individuals may have different taste for work and desire different numbers of hours, even if they have the same values on the explanatory variables. $\varepsilon_{i}$ is an additive measurement error. With the error structure adopted here, $\varepsilon_{i}$ can also contain an optimization error term that arises because individuals cannot fine-tune their hours of work. This accounts for the fact that some options for hours of work may not be available for some individuals. $\pi_{i}^{*}$ is desired hours of work when there are no hour restrictions due to job availability. When there is no censoring, as in Blomquist and Newey (2002), observed hours of work $h_{i}=h_{i}{ }^{*}$. The specification with two error terms adopted here and by Blomquist and Newey (2002) is the same as in the Hausman approach. A consequence is that observed hours of work need not be the outcome that would have maximized utility had there been no hour restrictions.

I handle censoring in a latent variable framework, treating $h_{i}{ }^{*}$ as latent hours of work. Observed hours of work $h_{i}$ and the observed participation (dummy) $D\left(h_{i}>0\right)$ become:

$$
h_{i}=\left\{\begin{array}{ll}
h_{i}{ }^{*} \\
0
\end{array} \text { and } D\left(h_{i}>0\right)= \begin{cases}1 & \text { if } h_{i}{ }^{*}>0 \Leftrightarrow \varepsilon_{i}>-\pi_{i}{ }^{*} \\
0 & \text { if } h_{i}{ }^{*} \leq 0 \Leftrightarrow \varepsilon_{i} \leq-\pi_{i}{ }^{*} .\end{cases}\right.
$$

Unlike in many binary outcome models, the latent variable in this setup has an economic interpretation. It is observed hours when there is no censoring. In such a world, negative hours would imply that the individual purchases leisure at her first-segment wage rate. ${ }^{1}$

\footnotetext{
${ }^{1}$ There is also a maximum hour censoring as time endowment is limited. This is ignored as the probability of being located there is zero in practice. Accounting for the maximum hour censoring would adjust the derived expressions very little and would not change the empirical specifications.
} 
The joint distribution of $v_{i}$ and $\varepsilon_{i}$ is denoted by $f\left(v_{i}, \varepsilon_{i}\right)$. I assume that $v_{i}$ is statistically independent of $\boldsymbol{x}_{\boldsymbol{i}}$ and $\boldsymbol{z}_{\boldsymbol{i}}$. This is appropriate when the tax system and the controls are exogenous and when the remaining unobserved heterogeneity is not determining gross income variables. Additionally, I assume that $E\left(\varepsilon_{i} \mid \boldsymbol{x}_{i}, z_{i}\right)=0$ and that $\pi_{i}{ }^{*}$ is strictly increasing in $v_{i}$. These assumptions are similar to those made by Blomquist and Newey (2002) except for the addition of $\boldsymbol{z}_{\boldsymbol{i}}$. The independence between $v_{i}$ and $\boldsymbol{x}_{\boldsymbol{i}}$ that they require is relaxed to conditional independence given $\boldsymbol{z}_{i}$. No distributional assumptions are made about the error terms, unlike those needed for ML methods, which make the estimation more robust to misspecification. The cost is an efficiency loss when the distributional assumptions hold and also that parameters of $f\left(v_{i}, \varepsilon_{i}\right)$ cannot be retrieved. From here on, the $i$-index is usually left out.

The tax and transfer system creates nonlinear budget sets containing piecewise-linear segments in the income-hour space. In a static framework, individuals consume all their income and are located on the budget frontier. A budget set with $J$ segments indexed by $j$ can be fully described by the slopes $w_{j}$ and the intercepts $y_{j}$ of each of the extended segments and the end point $l_{j}$ of all but the last segment; i.e., $\boldsymbol{x}=\left(w_{1 \ldots} w_{J} y_{1 \ldots} y_{J} l_{1 \ldots} l_{J-1}\right)^{\prime}$. If the budget frontier is continuous, then $l_{j}=\left(y_{j}-y_{j+1}\right) /\left(w_{j+1}-w_{j}\right)$. The slope of a segment is the net (marginal) wage rate (after all taxes), and the intercept of the extended segment is the (net marginal) virtual income (after all taxes) of that segment. $y_{1}$ is the unearned income. In a secondary-earner framework, the spouse's income is part of the unearned income. A three-segment piecewiselinear convex budget set is shown in Figure 1.

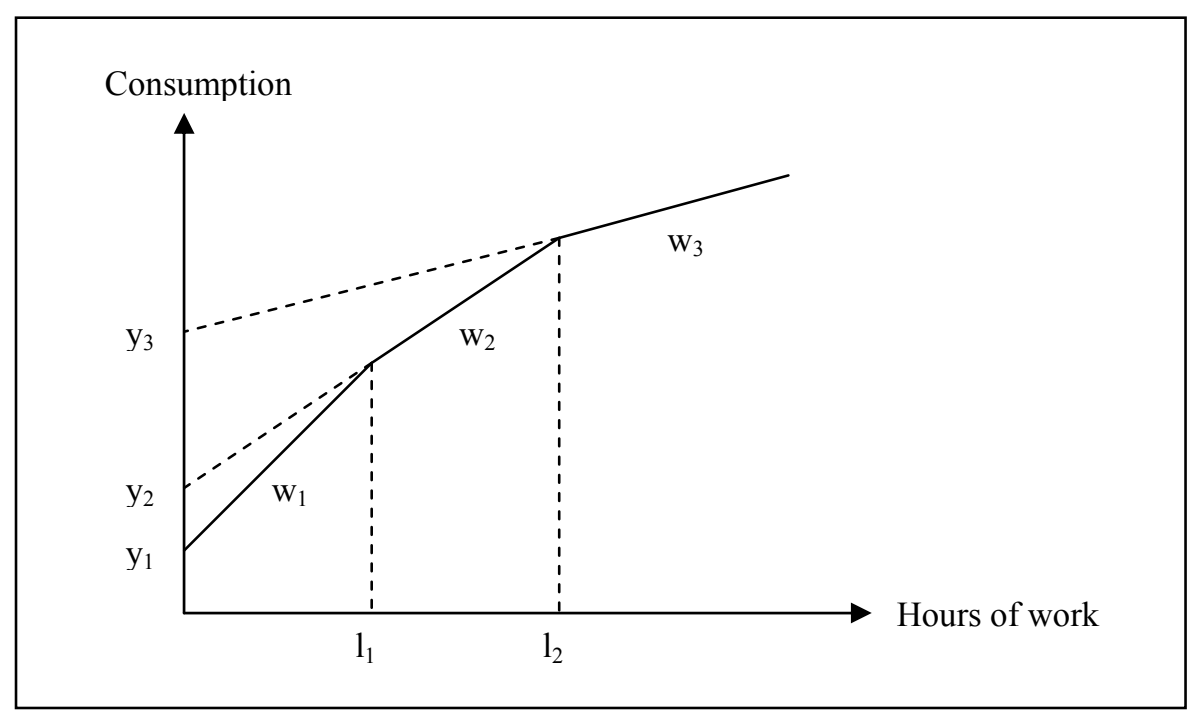

Figure 1. Three-segment piecewise-linear convex budget set 
Desired hours of work on a budget set that is a linearization of the $j^{\text {th }}$ segment is denoted $\pi_{j}(v)=\pi\left(w_{j}, y_{j}, z, v\right)$, and the inverse of $\pi_{j}(v)$ is denoted $\pi_{j}^{-1}(l)$. Under utility maximization subject to piecewise-linear convex budget constraints, individuals desire to locate on the segments and kinks according to:

$$
\pi^{*}= \begin{cases}\pi_{1}(v) & \text { if }-\infty \leq v \leq \pi_{1}^{-1}\left(l_{1}\right) \\ \pi_{j}(v) & \text { if } \pi_{j}^{-1}\left(l_{j-1}\right) \leq v \leq \pi_{j}^{-1}\left(l_{j}\right) \text { where } j=2, \ldots, J-1 \\ \pi_{J}(v) \text { if } \pi_{J}^{-1}\left(l_{J-1}\right) \leq v \leq \infty & \\ l_{j} & \text { if } \pi_{j}^{-1}\left(l_{j}\right) \leq v \leq \pi_{j+1}{ }^{-1}\left(l_{j}\right) \text { where } j=1, \ldots, J-1\end{cases}
$$

Expected hours of work and the participation probability are derived after summing terms on each of the segments and kinks, and are reported in Propositions 1 and 2.

PROPOSITION 1. When there is censoring at zero hours of work, expected hours of work under utility maximization subject to piecewise-linear convex budget constraints conditional on income and control variables can be written as

$$
\begin{aligned}
& E(h \mid \boldsymbol{x}, \boldsymbol{z})=\bar{\pi}\left(w_{J}, y_{J}, \boldsymbol{z}\right)+\sum_{j=1}^{J-1}\left[\mu\left(w_{j}, y_{j}, l_{j}, \boldsymbol{z}\right)-\mu\left(w_{j+1}, y_{j+1}, l_{j}, \boldsymbol{z}\right)\right] \\
& \text { where } \bar{\pi}\left(w_{J}, y_{J}, \boldsymbol{z}\right)=\int_{-\infty}^{\infty} \int_{-\pi\left(w_{J}, y_{J}, z, v\right)}^{\infty}\left[\pi\left(w_{J}, y_{J}, \boldsymbol{z}, v\right)+\varepsilon\right] f(v, \varepsilon) d \varepsilon d v, \\
& \mu\left(w_{j}, y_{j}, l_{j}, \boldsymbol{z}\right)= \\
& \int_{-\infty}^{\pi^{-1}\left(w_{j}, y_{j}, l_{j}, z\right)}\left\{\int_{-\pi\left(w_{j}, y_{j}, z, v\right)}^{\infty}\left[\pi\left(w_{j}, y_{j}, \boldsymbol{z}, v\right)+\varepsilon\right] f(v, \varepsilon) d \varepsilon-\int_{-l_{j}}^{\infty}\left[l_{j}+\varepsilon\right] f(v, \varepsilon) d \varepsilon\right\} d v .
\end{aligned}
$$

Proof. See Appendix A.

PROPOSITION 2. When there is censoring at zero hours of work, the participation probability under utility maximization subject to piecewise-linear convex budget constraints conditional on income and control variables can be written as

$$
\begin{aligned}
& \operatorname{Pr}(h>0 \mid \boldsymbol{x}, \boldsymbol{z})=\bar{\pi}^{P}\left(w_{J}, y_{J}, \boldsymbol{z}\right)+\sum_{j=1}^{J-1}\left[\mu^{P}\left(w_{j}, y_{j}, l_{j}, \boldsymbol{z}\right)-\mu^{P}\left(w_{j+1}, y_{j+1}, l_{j}, \boldsymbol{z}\right)\right] \\
& \text { where } \bar{\pi}^{P}\left(w_{J}, y_{J}, \boldsymbol{z}\right)=\int_{-\infty}^{\infty} \int_{-\pi\left(w_{J}, y_{J}, z, v\right)}^{\infty} f(v, \varepsilon) d \varepsilon d v, \\
& \mu^{P}\left(w_{j}, y_{j}, l_{j}, \boldsymbol{z}\right)=\int_{-\infty}^{\pi^{-1}\left(w_{j}, y_{j}, l_{j}, z\right)} \int_{-\pi\left(w_{j}, y_{j}, z, v\right)}^{-l_{j}} f(v, \varepsilon) d \varepsilon d v .
\end{aligned}
$$

Proof. See Appendix A. 
Equations (5) and (6) represent expected hours of work and the participation probability in terms of the labor supply in a budget set that is a linearization of the last segment and a correction term for nonlinearities. The expressions have the same structure and are identical to Blomquist and Newey's (2002) formula for expected hours when there is no censoring with respect to dimensionality, and the expressions can be estimated nonparametrically along their approach. Hence, naively applying their formula for expected hours in the presence of censoring would accidentally produce the same empirical specification as when applying the proper censoring-adjusted formula. This result is, however, not trivial; Proposition 1 is needed to realize this. Expected hours collapse to their simpler expression when there is no censoring and there are no control variables. The linear term has a dimensionality equal to two plus the number of controls. The correction term has a particular difference form in which the dimensionality is three plus the number of controls independent of the number of segments in the budget set. Blomquist and Newey (2002) show that the accuracy in estimating this term is independent of the number of segments.

The way to account for the censoring outlined here does not require additional distributional assumptions, in contrast to parametric methods. Neither does it require exclusion restrictions, in contrast to usual nonparametric methods. Responses on the participation and hours margins can also be sorted out. The straightforward structural extension preserves all features of the original Blomquist and Newey (2002) method, including asymptotic properties, and feasibility and simplicity to estimate nonparametrically.

It is however impossible to distinguish parameters belonging to the linear budget set term from those belonging to the correction term since some explanatory variables enter both. It is also impossible to identify parameters belonging to desired hours from the measurement error for the same reason. We can, however, consistently identify the effects of changes in explanatory variables, such as the effect of budget set changes on the labor supply. A basic requirement is that the budget sets are known and characterized properly.

\section{Empirical Specification and Estimation}

Blomquist and Newey (2002) estimate expected hours of work nonparametrically by approximating each term with power series regressors and then using OLS. Power series are convenient because additivity and equality constraints are easily incorporated into the estimation by excluding interaction regressors and by imposing equality of coefficients. OLS is at- 
tractive in terms of implementational simplicity and computational time. This approach can also be used to estimate expected hours of work and the participation probability when there is censoring. In fact, the empirical specification becomes similar, since all these entities result in expressions with an identical structure. Differences in the sets of regressors for the different entities are due to the empirical selection of regressors that is described later in this section.

The dimensionality of each term is low when control variables are omitted, as they are in Blomquist and Newey (2002). The potential number of approximating regressors for each term increases exponentially with the number of controls due to new combinations of interaction regressors. Feasible estimation therefore requires parsimony in the number of controls that are included. ${ }^{2}$ However, to reasonably argue that the remaining unobserved heterogeneity is independent of the budget set requires that variables that may determine gross income variables (before any taxes) and taste for work are included as controls. Based on these considerations, I include three demographic control variables: age $a$, number of children $c$, and years of education (including vocational training) $e$. To account for additional unobserved timevarying heterogeneity, such as attitudes, technology, and macroeconomics, I also include time dummies, Y80, Y90, and Y99, for each of the three latest years, 1980, 1990, and 1999, in the data. The first year, 1973, is left out to avoid multicollinearity. Therefore, $z=($ a c e Y80 Y90 Y99)'. ${ }^{3}$

Using $\sim$ to denote approximation of terms with a vector, the terms in expected hours of work in equation (5) in Proposition 1 are approximated as:

$$
\begin{aligned}
& \bar{\pi}\left(w_{J}, y_{J}, \boldsymbol{z}\right) \sim \boldsymbol{p}^{\boldsymbol{M}}(\boldsymbol{x}, \boldsymbol{z})=\left[p_{1}(\boldsymbol{x}, \boldsymbol{z}) \ldots p_{M}(\boldsymbol{x}, \boldsymbol{z})\right] \\
& \text { where } p_{m}(\boldsymbol{x}, \boldsymbol{z})=a^{m_{1}(m)} c^{m_{2}(m)} e^{m_{3}(m)} Y 80^{m_{4}(m)} Y 90^{m_{5}(m)} Y 99^{m_{6}(m)} w_{J}^{m_{7}(m)} y_{J}^{m_{8}(m)} \\
& \sum_{j=1}^{J-1}\left[\mu\left(w_{j}, y_{j}, l_{j}, \boldsymbol{z}\right)-\mu\left(w_{j+1}, y_{j+1}, l_{j}, \boldsymbol{z}\right)\right] \\
& \sim \boldsymbol{q}^{\boldsymbol{N}}(\boldsymbol{x}, \boldsymbol{z})=\left[q_{1}(\boldsymbol{x}, \boldsymbol{z}) \ldots q_{N}(\boldsymbol{x}, \boldsymbol{z})\right] \text { where } q_{n}(\boldsymbol{x}, \boldsymbol{z})= \\
& a^{n_{1}(n)} c^{n_{2}(n)} e^{n_{3}(n)} Y 80^{n_{4}(n)} Y 90^{n_{5}(n)} Y 99^{n_{6}(n)} \sum_{j=1}^{J-1} l_{j}^{n_{7}(n)}\left(w_{j}^{n_{8}(n)} y_{j}^{n_{9}(n)}-w_{j+1}^{n_{8}(n)} y_{j+1}^{n_{9}(n)}\right) .
\end{aligned}
$$

\footnotetext{
${ }^{2}$ A method to handle a larger number of control variables is to use an index specification $\pi_{j}=\pi\left(y_{j}, w_{j}, \gamma^{\prime} z, v\right)$ along the lines of that used by Ichimura (1993), which would only add one dimension to each term independent of the number of controls. I find such a specification too restrictive.

${ }^{3}$ Other potential controls available are: socio-economic, metropolitan area, municipality, county, and individual dummies/fixed effects. Many of these variables are problematic to include because they take away a lot of the variation in the income variables, decrease the degrees of freedom substantially, or cannot be estimated consistently due to too little variation or too few observations.
} 
The approximation is similar for the terms in the participation probability in equation (6) in Proposition 2. Capital letters denote the number of approximating terms, and lowercase letters index them. Numbered letters are integer power order functions. The empirical specification of hours of work, which is estimated with OLS, is

$$
h=\beta_{0}+\boldsymbol{\beta}_{p}{ }^{\prime} \boldsymbol{p}^{M}(\boldsymbol{x}, \boldsymbol{z})+\boldsymbol{\beta}_{q}{ }^{\prime} \boldsymbol{q}^{N}(\boldsymbol{x}, \boldsymbol{z})+\xi,
$$

where $\beta_{0}$ is a constant and $\xi$ is an error term. For participation, the dependent variable $h$ is replaced with $D(h)$.

Many potential approximating regressors in the approximating vectors are perfectly collinear and need to be excluded. Potential first and second order regressors in $\boldsymbol{p}^{\boldsymbol{M}}(\boldsymbol{x}, \boldsymbol{z})$ are $a$, $a^{2}, c, c^{2}, e, e^{2}, a c, c e, Y 80, Y 90, Y 99, a Y 80, a Y 90, a Y 99, c Y 80, c Y 90, c Y 99, e Y 80, e Y 90, e Y 99$, $w_{J}, y_{J}, w_{J} y_{J}, w_{J}^{2}, y_{J}^{2}, a w_{J}, a y_{J}, c w_{J}, c y_{J}, e w_{J}, e y_{J}, Y 80 w_{J}, Y 80 y_{J}, Y 90 w_{J}, Y 90 y_{J}, Y 9 q_{J}$, and $Y 99 y_{J}$. Similarly, $\boldsymbol{q}^{N}(\boldsymbol{x}, \boldsymbol{z})$ could contain regressors such as $\Delta w, \Delta y, \Delta w y, \Delta w^{2}, \Delta y^{2}, l \Delta y, a \Delta w$, $a \Delta y, c \Delta w, c \Delta y, e \Delta w, e \Delta y, Y 80 \Delta w, Y 80 \Delta y, Y 90 \Delta w, Y 90 \Delta y, Y 99 \Delta w$, and $Y 99 \Delta y$, where $a^{o_{1}} c^{o_{2}} e^{o_{3}} Y 80^{o_{4}} Y 90^{o_{5}} Y 99^{o_{6}} l^{o_{7}} \Delta w^{o_{8}} y^{o_{9}}=a^{o_{1}} c^{o_{2}} e^{o_{3}} Y 80^{o_{4}} Y 90^{o_{5}} Y 99^{o_{6}} \sum_{j=1}^{J-1} l_{j}^{o_{7}}\left(w_{j}^{o_{8}} y_{j}^{o_{9}}-w_{j+1}^{o_{8}} y_{j+1}^{o_{9}}\right)$.

For non-working individuals, gross wage rates are imputed by separately estimating a gross wage rate function with the working individuals for each of the four cross-sections. ${ }^{4}$ There is no need to account for selection in the gross wage rate function, as the estimates here are only used for prediction and not for causal inference. The regressions give coefficients of determination around 0.3 . The gross wage rate and the unearned income are used together with the relevant tax and transfer system to construct the net wage rates and the virtual incomes needed to estimate the labor supply function.

Flexible selection of the number as well as the power order of the approximating regressors is the nonparametric component in the estimation of equation (9). I use the same crossvalidation criteria that Blomquist and Newey (2002) use to select the exact set of regressors:

$$
C V=1-\frac{S S E}{\sum_{i}\left[h_{i}-\hat{h}_{i}\right]^{2}}, \text { where } S S E=\sum_{i} \frac{\left[h_{i}-\hat{h}_{i}^{-i}\right]^{2}}{\left[1-\boldsymbol{R}^{\prime}\left(\boldsymbol{R}^{\prime} \boldsymbol{R}\right)^{-1} \boldsymbol{R}_{i}\right]^{2}} .
$$

$S S E$ is the sum of squares of the forecast errors normalized with the inverse of squares of one minus the respective diagonal element in the projection matrix $\boldsymbol{R}^{\prime}\left(\boldsymbol{R}^{\prime} \boldsymbol{R}\right)^{-1} \boldsymbol{R}$, where $\boldsymbol{R}$ is the

\footnotetext{
${ }^{4}$ A quadratic in age, number of children, and education years, as well as education level, socio-economic, county, and metropolitan area dummies are used as regressors in these regressions. The goal with this regression is goodness of fit, and I therefore choose to use an extensive set of explanatory variables.
} 
matrix of all regressors. The forecast of $i, \hat{h}_{i}^{-i}$ is the predicted value using all but the $i^{\text {th }}$ observation. The division of $S S E$ with the sample sum of squares of $h$ makes the crossvalidation value invariant to the scale of $h$. Maximizing $C V$ minimizes the asymptotic meansquare error and the bias goes to zero at the same rate as the standard deviation according to Andrews (1991). More terms are needed for a faster convergence rate.

Since there are a lot of combinations of regressors, finding the global $\mathrm{CV}$ maximum is not easy. I follow some simple principles to make the selection process tractable. To begin with, first-order approximating regressors are included. Additional groups of regressors are then added, one group at a time, until the $C V$ maximum clearly seems to be passed. I consecutively add the group whose inclusion maximizes $C V$ among a pool of groups. The pool contains groups with all the lowest-order terms that have not yet been added. The pool can be expanded with higher-order groups each time a group has been added. A composite regressor such as $c^{2} \Delta w^{2}$ cannot be added before its subfactors $c, c^{2}, \Delta w, \Delta w^{2}, c \Delta w, c^{2} \Delta w$, and $c \Delta w^{2}$ have been added. Each group contains regressors of similar type and order. An example of a group is $a c, a e$, and $c e$; another example is $w_{J}^{2}$ and $\Delta w^{2}$.

The flexibility advantage of nonparametrics is magnified when there are control variables. The impacts of the income and control variables are flexible by themselves and when interacted. This allows both the responsiveness of the labor supply to budget set changes and the impact of demographics to vary over time. The flexibility is, however, demanding on the data. Demographic controls and time dummies alone remove a lot of the variation between individuals and time periods, respectively. The remaining variation in gross income variables, such as the gross wage and the unearned income, provides some individual variation useful for identification. Tax reforms that have different impacts on individuals with different budget sets provide a crucial source of variation, as in natural experiment-based approaches. Even some of this variation could, however, be removed by interactions between demographic variables and time dummies, leaving much of the identification to the specific structure of the budget set imposed by utility maximization and the selection criterion.

The results from labor supply estimations are often summarized in terms of elasticity measures that reflect the responsiveness of labor supply. Nevertheless, caution is needed in interpreting and comparing such figures. Elasticities typically vary enormously with the point of evaluation, which is illustrated by Blomquist and Hansson-Brusewitz (1990).

Another caveat is that there is not a single net wage rate or virtual income when the budget set is nonlinear. It is therefore artificial to speak of the effect of a change in the net 
wage or virtual income on labor supply, even if this may be natural from the point of view of the empirical specification in many methods. It is also problematic in most cases to try to distinguish the effects of the net wage or the virtual income on a specific segment from those on another segment, as changing the net wage or virtual income on one segment usually also automatically changes those on others. Most authors, including Blomquist and Newey (2002), report and interpret elasticities as the responsiveness in linear budget sets where there are clearly a single net wage and virtual income. Some extrapolation is, however, required either in the construction or in the interpretation of such a measure, as there are no linear budget sets in the data. The measure may therefore not be very informative.

Despite these shortcomings, it is common practice and convenient to report results in terms of elasticities. In the present method, there is also such a need, since the parameters themselves are completely uninformative. However, it is not clear how to construct elasticities, since variables characterizing the whole budget set are used in the estimation. To be able to compare with results in other papers, I first follow Blomquist and Newey (2002) and use the regression estimates to construct the uncompensated wage elasticity of labor supply $E l_{h \mid w}$ and the income elasticity of labor supply $E l_{h \mid w}$ for a linear budget set with $\boldsymbol{x}=\boldsymbol{x}_{\text {linear }}=\boldsymbol{x}(w, y)=\left(w_{l}=w_{\ldots} w_{J}=w y_{l}=y_{\ldots} y_{J}=y\right)^{\prime}$ :

$$
\begin{aligned}
& \left.E l_{h \mid w}\left(\boldsymbol{x}=\boldsymbol{x}_{\text {linear }}, \boldsymbol{z}\right) \equiv \frac{w}{h} \frac{\partial h}{\partial w^{*}}\left(w_{1}=w^{*}, \ldots, w_{J}=w^{*}\right)\right|_{w^{*}=w}, \\
& \left.E l_{h \mid y}\left(\boldsymbol{x}=\boldsymbol{x}_{\text {linear }}, \boldsymbol{z}\right) \equiv \frac{y}{h} \frac{\partial h}{\partial y^{*}}\left(y_{1}=y^{*}, \ldots, y_{J}=y^{*}\right)\right|_{y^{*}=y},
\end{aligned}
$$

Depending on the kind of comparison for which the elasticities are used, $h$ is sometimes chosen, and sometimes functions of $\boldsymbol{x}$ and $\boldsymbol{z}$. Note that there is only one virtual income, which is the unearned income in linear budget sets. Standard errors are constructed using the bootstrap.

Due to the problems with these elasticities, I find the main use of the labor supply regression estimates to be predicting Mongrel labor supply (defined by Blomquist and HanssonBrusewitz, 1990), which is the labor supply given explanatory variables for a tax system at hand. By letting certain non-tax variables vary, Mongrel labor supply functions for a tax system can be constructed. Different tax systems can be evaluated by comparing their Mongrel labor supply functions. For a given sample, the sample statistics of predicted Mongrel labor supply also provide an aggregate picture. Important in these simulations is that the simulated budget sets need to have equivalents in the data used for estimation to avoid extrapolation. 
To obtain a more relevant and less problematic elasticity summary measure, I propose a simulation-based tax-system and tax-reform tied aggregate Mongrel elasticity measure for a given population at a certain point in time. A certain tax system can be described by the tax matrix $\boldsymbol{T}=\left(\boldsymbol{\tau}_{r} \tau_{b}\right)$, where $\boldsymbol{\tau}_{r}$ represents the tax rates and $\boldsymbol{\tau}_{\boldsymbol{b}}$ represents the tax brackets. A population at a certain point in time can be described by the gross incomes $\boldsymbol{x}_{\boldsymbol{G}}$, demographics and time period $\boldsymbol{Z}$, and the budget sets $\boldsymbol{X}\left(\boldsymbol{T}, \boldsymbol{x}_{G}, \boldsymbol{Z}\right)$, where the population vector and matrices are obtained by stacking the individual equivalents. $i$ is used to index the elements in the vectors and matrices for an individual. A tax reform $\boldsymbol{\Delta T}$ induces budget set changes $\boldsymbol{\Delta} \boldsymbol{X}\left(\boldsymbol{\Delta} \boldsymbol{T}, \boldsymbol{x}_{\boldsymbol{G}}, \boldsymbol{Z}\right)$. Each aggregate elasticity is then a function of $\boldsymbol{T}, \boldsymbol{x}_{\boldsymbol{G}}, \boldsymbol{Z}$, and $\boldsymbol{\Delta T}$.

To simplify notation, the subindex pre denotes the pre-reform situation with budget sets $\boldsymbol{X}$, and the subindex post denotes the post-reform situation with budget sets $\boldsymbol{X}+\boldsymbol{\Delta} \boldsymbol{X}$. The aggregate Mongrel wage and income elasticities of labor supply are then $\overline{E l}_{h \mid w, p r e}$ and $\overline{E l}_{h \mid y, p r e}$. Another interesting elasticity is the aggregate Mongrel net-of-tax elasticity of taxable income $\overline{E l}_{Y \mid 1-\bar{\tau}, \text { pre }}$, where $Y$ is taxable income and $\bar{\tau}$ is the average tax rate. The elasticities are constructed as:

$$
\begin{aligned}
& \overline{E l}_{h \mid w, p r e}\left[\boldsymbol{\Delta} \boldsymbol{X}\left(\boldsymbol{\Delta} \boldsymbol{y}_{1}=0\right)\right] \equiv \\
& \lim _{\boldsymbol{\Delta T} \rightarrow 0} \frac{\sum_{i}\left(1-\bar{\tau}_{h>0, p r e}\right) w_{G, i}}{\sum_{i} \hat{h}_{h>0, p r e}} \frac{\sum_{i} \hat{h}_{i, p o s t}-\sum_{i} \hat{h}_{i, p r e}}{\sum_{i}\left[1-\bar{\tau}_{h>0, p o s t}\left(\hat{\boldsymbol{h}}=\hat{\boldsymbol{h}}_{\text {pre }}\right)\right] w_{G, i}-\sum_{i}\left(1-\bar{\tau}_{h>0, p r e}\right) w_{G, i}} \\
& \overline{E l}_{h \mid y, p r e}[\boldsymbol{\Delta} \boldsymbol{X}(\boldsymbol{\Delta} \boldsymbol{W}=0)] \equiv \lim _{\boldsymbol{\Delta} \boldsymbol{T} \rightarrow 0} \frac{\sum_{i} y_{1, i, p r e}}{\sum_{i} \hat{h}_{i, p r e}} \frac{\sum_{i} \hat{h}_{i, p o s t}-\sum_{i} \hat{h}_{i, p r e}}{\sum_{i} y_{1, i, p o s t}-\sum_{i} y_{1, i, p r e}} \\
& \overline{E l}_{Y \mid(1-\bar{\tau}), p r e}(\boldsymbol{\Delta X}) \equiv \lim _{\boldsymbol{\Delta} \boldsymbol{T} \rightarrow 0} \frac{\sum_{i}\left(1-\bar{\tau}_{\text {pre }}\right)}{\sum_{i} \hat{Y}_{i, p r e}} \frac{\sum_{i} \hat{Y}_{i, p o s t}-\sum_{i} \hat{Y}_{i, p r e}}{\sum_{i}\left[1-\bar{\tau}_{p o s t}\left(\hat{\boldsymbol{h}}=\hat{\boldsymbol{h}}_{\text {pre }}\right)\right]-\sum_{i}\left(1-\bar{\tau}_{\text {pre }}\right)}
\end{aligned}
$$

where $\hat{Y}_{i} \equiv w_{G, i} \hat{h}\left(\boldsymbol{x}_{\boldsymbol{i}}, \boldsymbol{z}_{\boldsymbol{i}}\right)+y_{1, i}$

$$
\text { and } \bar{\tau}_{h>0} \equiv \frac{\sum_{i} \hat{T}_{h>0}\left(\hat{h}_{i}, \boldsymbol{x}_{\boldsymbol{i}}, \boldsymbol{z}_{\boldsymbol{i}}\right)}{\sum_{i} w_{G, i} \hat{h}\left(\boldsymbol{x}_{\boldsymbol{i}}, \boldsymbol{z}_{\boldsymbol{i}}\right)}, \bar{\tau} \equiv \frac{\sum_{i}\left[\hat{T}_{h>0}\left(\hat{h}_{i}, \boldsymbol{x}_{\boldsymbol{i}}, \boldsymbol{z}_{\boldsymbol{i}}\right)+\hat{T}_{1, i}\right]}{\sum_{i}\left[w_{G, i} \hat{h}\left(\boldsymbol{x}_{\boldsymbol{i}}, \boldsymbol{z}_{\boldsymbol{i}}\right)+y_{1, i}\right]}
$$

$T\left(\boldsymbol{x}_{i}, z_{i}\right)$ is the tax revenue function, and $\bar{\tau}(*)$ is the average tax function. Unearned incomes are held constant when calculating the uncompensated wage elasticity; i.e., the unearned in- 
come change subvector $\Delta \boldsymbol{y}_{1}=\boldsymbol{0}$ in the matrix $\Delta X$. The idea is to exclude pure income effects. Net wages rates are instead held constant; i.e., the net wage rate submatrix $\boldsymbol{\Delta} \boldsymbol{W}=\boldsymbol{0}$ in the matrix $\boldsymbol{\Delta X}$. The idea is to study pure income effects due to changes in unearned incomes and to allow only changes in virtual incomes and kinks that accompany the unearned income changes.

The tax rate change used for denomination is the average tax with labor supply fixed to the pre-reform level described; i.e., $\hat{\boldsymbol{h}}=\hat{\boldsymbol{h}}_{\text {pre }}=\hat{\boldsymbol{h}}(\boldsymbol{X}, \boldsymbol{Z})$. Averaging is a convenient way to summarize nonlinear tax rates in a single conceivable rate, which, in contrast to the marginal rate, is not directly plagued by behavioral adjustments. Note that for a given marginal tax change in a segment, the average tax changes much less. Fixing labor supply is necessary to clean out behavioral effects of reforms on tax rates. The tax reform matrix approaches zero in the sense that all elements in it are shrunk keeping the ratio between them constant.

Finally, the tax elasticity of net government revenues can be constructed as

$$
\begin{aligned}
& \overline{E l}_{T \mid \tau, p r e}(\boldsymbol{\Delta X}) \equiv \lim _{\boldsymbol{\Delta} \boldsymbol{X} \rightarrow 0} \frac{\sum_{i} \bar{\tau}_{i, \text { pre }} \sum_{i} \hat{T}_{i, p o s t}-\sum_{i} \hat{T}_{i, p r e}}{\sum_{i} \bar{\tau}_{i, p o s t}-\sum_{i} \bar{\tau}_{i, p r e}} \\
& =\lim _{\boldsymbol{\Delta} \boldsymbol{T} \rightarrow 0} \frac{\sum_{i} \hat{T}_{i, p o s t}-\sum_{i} \hat{T}_{i, p r e}}{\sum_{i} \hat{T}_{i, p o s t}\left(\hat{h}_{i, p o s t}=\hat{h}_{i, p r e}\right)-\sum_{i} \hat{T}_{i, p r e}}
\end{aligned}
$$

For a concave Laffer curve, this elasticity is positive up the revenue maximum and negative after that.

\section{Data and Summary Statistics}

In the estimation of female labor supply, I use data from 1973, 1980, 1990, and 1999 from the Swedish "Level of Living" (LNU) surveys undertaken in 1974, 1981, 1991, and 2000. The Swedish National Data Service (SND) and the Institute of Social Research (SOFI) at Stockholm University have provided the survey data and data on taxation and social security. The surveys cover around 6,000 individuals for each wave and make up a random sample con-

\footnotetext{
${ }^{5}$ It is possible to construct individual elasticity measures without aggregating over the population. For such elasticities the following relationship holds: $E l_{T \mid 1-\bar{\tau}, \text { pre }}=1-\frac{\bar{\tau}_{i, p r e}}{1-\bar{\tau}_{i, p r e}} E l_{Y \mid 1-\bar{\tau} \text {,pre }}$. The average of the individual elasticities usually differs from the corresponding aggregate elasticity.
} 
sisting of $1 / 1,000^{\text {th }}$ of the Swedish population in the age span of 18 to 75 years old. It is a rotating panel where about 2,500 individuals appear in all four surveys. The overall response rate in the four surveys is 81.6 percent.

The data from 1973, 1980, and 1990 have previously been used in labor supply studies several times. Blomquist (1983), Blomquist and Hansson Brusewitz (1990), and Aronsson and Palme (1998) use the Hausman approach. Blomquist and Newey (2002) and Blomquist et al. (2001) use the same nonparametric approach as here, but they use it on married men. There are also a number of studies (Selén, 2005; Ljunge and Ragan, 2006; Hansson, 2007; Gelber, 2008; and Blomquist and Selin, 2009) that look at taxable income and the extensive reforms from 1980 to 1991 using register data and natural experiment or instrumental variables approaches.

It is common in the labor supply literature to select the sample quite heavily. One rationale is that different groups may have different responsiveness in labor supply. Another important reason is that it is difficult to characterize the budget sets of some groups of individuals satisfactorily. The selection made here is very similar to those made in the papers mentioned above, but still, it leaves out many groups that are also of great interest. I limit my sample to married or cohabiting women in the age span of 20 to 60 years old without small children (aged five years old or less). Pensioners, students, those with more than four weeks of sick leave, farmers, and self-employed are also excluded. The number of observations dropped in each step is reported in Table A1 in Appendix B. There remain 2,882 observations after the selection, 825 from 1973, 746 from 1980, 698 from 1990, and 613 from 1999.

The distribution of yearly hours of work in the sample is shown in Figure A1 in Appendix B. The four peaks correspond to individuals who do not work, work half-time, work 75 percent, and work full-time. Around 60 percent of the observations are clustered at these four locations.

For 1973, 1980, and 1990, I use the same stylized tax systems as used in Blomquist (1983), Blomquist and Hansson-Brusewitz (1990), Blomquist et al. (2001), and Blomquist and Newey (2002). The stylized tax system that I use for 1999 is described in Appendix C. These systems are used to construct the budget sets for the individuals; these budget sets are needed in the estimation. Some minor components of the tax systems have been left out due to the difficulty of modeling their effects on the budget sets and due to lack of relevance to the sample individuals.

The taxation of earned and capital incomes, including basic and capital deductions, is accounted for in the construction of the budget sets. Individual taxation was introduced in 
1970 prior to the sample period. Earned and capital incomes were jointly taxed until 1991. Payroll tax (PRT) and value added tax (VAT) are also accounted for. The social security and transfer system has become increasingly important over time. The two most important allowances for married couples, the child and housing allowances, are incorporated into the budget sets. In the selected sample, other programs, such as the parental cash benefit, various pensions, student finance, the sickness benefit, and social assistance, are of small or no importance and not considered.

The tax and transfer system contains minor non-convexities. As the labor supply functions are derived under the assumption of convex budget sets, I convexify the budget sets before estimation. Blomquist and Newey (2002) perform Monte Carlo simulations and statistical tests using non-convex budget set correction terms on a data set that is similar to the one used here. They find that there is little bias produced by imposing the convexity restrictions despite the presence of some non-convexities.

Summary statistics for some key variables are reported in Table 1. Means are reported without and standard deviations within parentheses. Yearly hours of work and hourly wage rates from the surveys are used. Measurement errors in the yearly hour dependent variable are accounted for in the estimation as long as they are not correlated with the explanatory variables, in which case there would be a bias. Using hourly wages from the surveys avoids the division bias reported by Eklöf and Sacklén (1999) that arises when using hourly wages calculated by dividing the yearly income by yearly hours. $\tau$ denotes marginal tax rates, and segments denotes the number of segments. Subscript actual denotes the actual segment on which individuals are located, 1 denotes the first segment, and $J$ denotes the last segment, where individuals at kinks are counted as facing the next segment. All income variables apply to the convexified budget sets. The wages reported are gross before payroll tax (pre-PRT) rates, whereas the tax rates and the virtual incomes are net after value added tax (post-VAT) figures.

Between 1973 and 1999, the labor supply of married women increased by 58.3 percent, from an average level of 55.0 to 87.1 percent of regular full-time work. This development nearly bridges the entire gap between them and their almost exclusively full-time working husbands. The rise is due to both increased participation as well as increased hours of work. However, there is also a positive trend in the average education, gross pre-PRT hourly wage, and age, as well as a negative trend in the average number of children. This co-variation over time indicates the need to include demographic variables or time dummies as controls. The variation in yearly hours of work and in the participation rate decrease over time as revealed by the standard deviations. 
Table 1. Summary statistics

\begin{tabular}{l|ccccc}
\hline Variables & Pooled & 1973 & 1980 & 1990 & 1999 \\
\hline $\mathrm{h}$ & 1,475 & 1,145 & 1,384 & 1,666 & 1,812 \\
& $(732)$ & $(826)$ & $(702)$ & $(597)$ & $(530)$ \\
$\mathrm{D}(\mathrm{h}>0)$ & 0.907 & 0.800 & 0.914 & 0.960 & 0.982 \\
& $(0.290)$ & $(0.400)$ & $(0.280)$ & $(0.196)$ & $(0.133)$ \\
$\mathrm{w}_{\text {pre-PRT }}$ & 116.268 & 83.478 & 104.571 & 144.158 & 142.875 \\
& $(42.978)$ & $(21.018)$ & $(26.286)$ & $(37.040)$ & $(49.844)$ \\
$\tau_{\text {actual }}$ & 0.600 & 0.476 & 0.611 & 0.706 & 0.632 \\
& $(0.144)$ & $(0.169)$ & $(0.117)$ & $(0.061)$ & $(0.063)$ \\
$\tau_{1}$ & 0.362 & 0.226 & 0.370 & 0.523 & 0.350 \\
& $(0.116)$ & $(0.019)$ & $(0.060)$ & $(0.053)$ & $(0.000)$ \\
$\tau_{\mathrm{J}}$ & 0.816 & 0.829 & 0.869 & 0.831 & 0.717 \\
& $(0.054)$ & $(0.000)$ & $(0.001)$ & $(0.006)$ & $(0.001)$ \\
$\mathrm{y}_{\text {actual }}$ & 157,942 & 131,231 & 165,698 & 169,589 & 171,192 \\
& $(74,917)$ & $(63,206)$ & $(61,493)$ & $(71,089)$ & $(96,528)$ \\
$\mathrm{y}_{1}$ & 142,173 & 117,343 & 149,009 & 155,577 & 152,010 \\
& $(73,025)$ & $(62,808)$ & $(59,025)$ & $(69,205)$ & $(94,375)$ \\
$\mathrm{y}_{\mathrm{J}}$ & 216,660 & 229,025 & 224,802 & 211,288 & 196,225 \\
& $(71,460)$ & $(60,777)$ & $(57,714)$ & $(67,994)$ & $(94,907)$ \\
segments & 9.759 & 8.995 & 16.429 & 5.725 & 7.263 \\
& $(4.194)$ & $(0.070)$ & $(1.258)$ & $(0.614)$ & $(0.778)$ \\
segments & 4.189 & 2.698 & 5.504 & 3.629 & 5.232 \\
& $(2.457)$ & $(1.620)$ & $(3.513)$ & $(1.076)$ & $(1.408)$ \\
$\mathrm{a}$ & 43.826 & 43.612 & 42.866 & 44.123 & 44.943 \\
& $(10.127)$ & $(10.438)$ & $(10.413)$ & $(9.654)$ & $(9.766)$ \\
$\mathrm{c}$ & 0.865 & 0.918 & 0.922 & 0.811 & 0.788 \\
& $(1.031)$ & $(1.052)$ & $(1.024)$ & $(1.000)$ & $(1.039)$ \\
$\mathrm{e}$ & 10.287 & 8.653 & 9.562 & 11.097 & 12.447 \\
& $(3.338)$ & $(2.856)$ & $(2.989)$ & $(3.158)$ & $(3.096)$ \\
\hline
\end{tabular}

Notes: Means are reported without and standard deviation reported within parentheses. $h$ is expressed in yearly hours, $w_{\text {pre-PRT }}, y_{\text {actual }}, y_{l}$, and $y_{J}$ in SEK (1999 price level, USD $1.0 \approx$ SEK 7.8), and $e$ in years.

The marginal tax rates in different parts of the budget sets are higher than usual for Sweden due to the inclusion of indirect taxes. The variation in average marginal tax rates between different parts of the budget set is quite large. The average marginal tax rate is lowest in the first segment, higher in the actual segment, and highest in the last segment, reflecting the progressivity of the tax systems. Among the different segments, the standard deviation is usually lowest for the last-segment marginal tax rates and highest for the actual segment. There is no clear trend over time, and it is difficult to rank the tax levels in the different years based on the statistics shown here.

Among the different segments, the last-segment marginal tax rate is the best indicator of the overall tax level, as the first-segment rate depends on capital income deductions and transfers, and as the actual rate depends on hours of work. The last-segment means are consistent with the general view that the overall tax level increased to a historical peak in 1980, de- 
creased to a historical low in 1991, and increased a little since then. The average number of segments partly reflects the complexity of the tax and transfer system and was highest in 1980 with an average of 16.2 segments. The average actual segment is quite low in relation to the average number of segments in 1973 and 1980, but it is higher in 1990 and 1999.

To get a more complete picture of the tax systems, marginal tax rates (including allowances and indirect taxation) at different gross pre-PRT earned income levels are plotted for an average individual experiencing each of the four different tax systems in Figure 2. There are only a few minor marginal tax rate changes after 500,000 SEK (1999 price level), at which the figure is truncated. We see that (marginal) tax rates increased at all income levels between 1973 and 1980, but decreased at all income levels between 1980 and 1990 and between 1990 and 1999. The decrease since 1980 is mainly at medium to high income levels, where tax rates have been reduced by over ten percentage points over large intervals. The 1999 tax system has higher tax rates at low income levels and lower tax rates at higher income levels compared to the 1973 tax system and is hence less progressive. The evolution of the tax system provides ample variation in budget sets, which is crucial for identification and simulations. The variation in tax systems also indicates that the results in this paper are of primary concern to high tax countries, but they are more general with respect to progressivity.

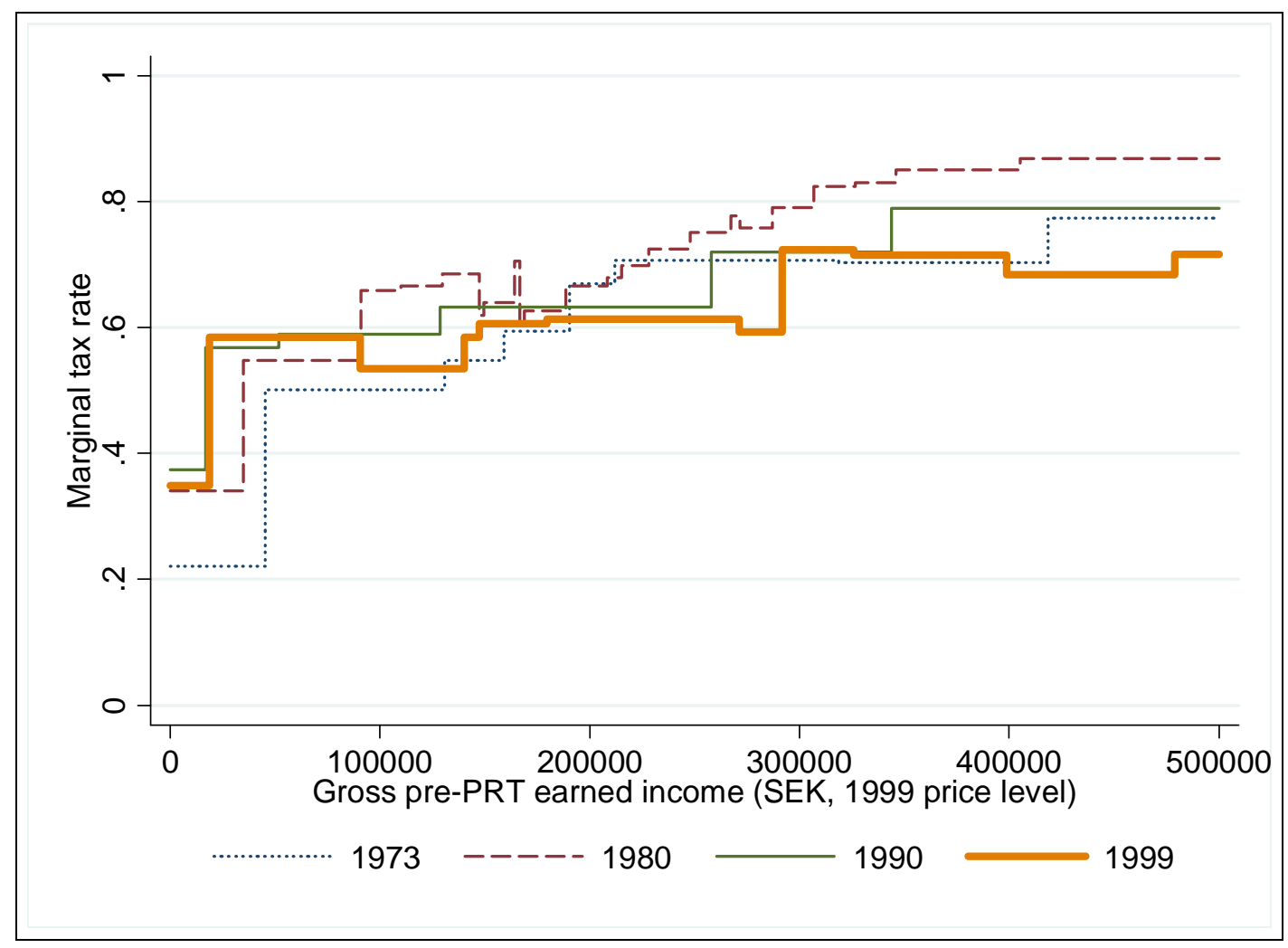

Figure 2. Marginal tax rates including allowances and indirect taxes 


\section{Estimation Results}

\subsection{Labor Supply Estimates}

The estimates for expected hours of work in equation (5) using the empirical specifications in equations (7) to (9) are reported in Table 2. Approximating regressors, the cross-validation value $(C V)$, and the uncompensated wage elasticity $E l_{h \mid w}\left(\boldsymbol{x}=\boldsymbol{x}_{\text {linear }}\right)$ and income elasticity $E l_{h \mid y}\left(\boldsymbol{x}=\boldsymbol{x}_{\text {linear }}\right)$ of labor supply for linear budget sets are reported. Additional regressors are added on top of the previous ones guided by the cross-validation criterion in equation (10). The elasticities are constructed from the regression estimates for a linear budget set at the pooled sample actual-segment mean according to equations (11) and (12). They are evaluated at the same fixed hours of work to enable comparison across specifications. The linear budget set elasticities are summary measures that should be used with caution as discussed in section three.

Table 2. Estimates for expected hours of work

\begin{tabular}{|c|c|c|c|c|}
\hline Specification & Additional terms & $\mathrm{CV}$ & $E l_{\mathrm{h} \mid \mathrm{w}}\left(\mathbf{x}=\mathbf{x}_{\text {linear }}\right)$ & $\mathrm{El}_{\mathrm{h} \mid \mathrm{y}}\left(\mathbf{x}=\mathbf{x}_{\text {linear }}\right)$ \\
\hline First order & constant, $\mathrm{w}_{\mathrm{J}}, \mathrm{y}_{\mathrm{J}}, \Delta \mathrm{w}, \Delta \mathrm{y}$ & 0.108 & $\begin{array}{c}0.491 \\
(0.056)\end{array}$ & $\begin{array}{c}-0.085 \\
(0.018)\end{array}$ \\
\hline Controls & a, c, e, Y1980, Y1990, Y1999 & 0.137 & $\begin{array}{c}0.416 \\
(0.098)\end{array}$ & $\begin{array}{l}-0.038 \\
(0.019)\end{array}$ \\
\hline \multirow{3}{*}{ Maximum } & $\mathrm{ac}, \mathrm{ae}, \mathrm{ce}, \mathrm{a}^{2}, \mathrm{c}^{2}, \mathrm{e}^{2}$ & 0.152 & $\begin{array}{c}0.446 \\
(0.098)\end{array}$ & $\begin{array}{l}-0.059 \\
(0.019)\end{array}$ \\
\hline & $1 \Delta \mathrm{y}$ & 0.164 & $\begin{array}{c}0.556 \\
(0.090)\end{array}$ & $\begin{array}{l}-0.063 \\
(0.019)\end{array}$ \\
\hline & ace, $a^{2} c, a^{2} e, a^{2}, c^{2} e, a e^{2}, c e^{2}$ & 0.157 & $\begin{array}{c}0.545 \\
(0.090)\end{array}$ & $\begin{array}{l}-0.062 \\
(0.019)\end{array}$ \\
\hline \multirow[t]{3}{*}{ Preferred } & $\mathrm{aw}_{\mathrm{J}}, \mathrm{cw}_{\mathrm{J}}, \mathrm{ew}_{\mathrm{J}}, \mathrm{a} \Delta \mathrm{w}, \mathrm{c} \Delta \mathrm{w}, \mathrm{e} \Delta \mathrm{w}$ & 0.147 & $\begin{array}{c}0.731 \\
(0.131)\end{array}$ & $\begin{array}{l}-0.059 \\
(0.018)\end{array}$ \\
\hline & $\mathrm{w}_{\mathrm{J}} \mathrm{y}_{\mathrm{J}}, \Delta \mathrm{w}_{\mathrm{J}} \mathrm{y}_{\mathrm{J}}$ & 0.135 & $\begin{array}{c}0.638 \\
(0.126)\end{array}$ & $\begin{array}{c}0.049 \\
(0.108)\end{array}$ \\
\hline & $\mathrm{w}_{\mathrm{J}}^{2}, \Delta \mathrm{w}^{2}$ & 0.122 & $\begin{array}{c}0.663 \\
(0.195)\end{array}$ & $\begin{array}{c}0.027 \\
(0.106)\end{array}$ \\
\hline
\end{tabular}

Notes: The elasticities are evaluated for a linear budget set at the pooled sample mean, i.e., $a=43.812$, $c=0.865, e=10.287, w=43.382, y=157,942$, and $h=1,475$. Standard errors are constructed using the bootstrap (1,000 replications) and are reported in parentheses.

First-order income variable regressors are included in First order. Demographic regressors and time dummies are added in Controls. Higher order regressors are then added. The crossvalidation value is maximized among the specifications tested in Maximum. The preferred specification from which subsequent simulations depart is reported in Preferred. The wage 
elasticity is positive and statistically significant. It decreases as first-order control regressors are added, and it usually increases as higher-order regressors are added. Accounting for demographics and time factors and using a flexible specification therefore matter. The sign of the income elasticity is ambiguous, and it is often statistically insignificant.

The cross-validation value increases with additional terms until it reaches 0.164 , which may, however, be a local maximum. For satisfactory convergence, the number of terms needs to be beyond the global cross-validation maximum. My preferred specification has many more terms to ensure this, but it still has a high cross-validation value, and it also has elasticities that are stable as more regressors are added. The wage elasticity is 0.731 , and the income elasticity is -0.059 . Both elasticities are statistically significant at the one percent level. The elasticities depend on demographics but not on other time factors in the preferred specification.

The estimates for the participation probability in equation (6) using the empirical specifications in equations (7) to (9) are reported in Table 3, which is organized like Table 2. The wage elasticity is positive and statistically significant. It increases as first-order control regressors are added, and it usually increases further as higher-order regressors are added. The sign of the income elasticity is ambiguous, and it is mostly statistically insignificant. My preferred wage elasticity is 0.177 , and income elasticity is insignificant. The elasticities depend again on demographics but not on other time factors in the preferred specification.

Table 3. Estimates for the participation probability

\begin{tabular}{|c|c|c|c|c|}
\hline Specification & Additional terms & $\mathrm{CV}$ & $\mathrm{El}_{\mathrm{h} \mid \mathrm{w}}\left(\mathbf{x}=\mathbf{x}_{\text {linear }}\right)$ & $\mathrm{El}_{\mathrm{h} \mid \mathrm{y}}\left(\mathbf{x}=\mathbf{x}_{\text {linear }}\right)$ \\
\hline First order & constant, $\mathrm{w}_{\mathrm{J}}, \mathrm{y}_{\mathrm{J}}, \Delta \mathrm{w}, \Delta \mathrm{y}$ & 0.035 & $\begin{array}{c}0.128 \\
(0.033)\end{array}$ & $\begin{array}{l}-0.030 \\
(0.012)\end{array}$ \\
\hline Controls & a, c, e, Y80, Y90, Y99 & 0.048 & $\begin{array}{l}0.156 \\
(0.050)\end{array}$ & $\begin{array}{l}-0.016 \\
(0.013)\end{array}$ \\
\hline & ac, ae, ce, $a^{2}, c^{2}, e^{2}$ & 0.061 & $\begin{array}{l}0.190 \\
(0.052)\end{array}$ & $\begin{array}{l}-0.032 \\
(0.013)\end{array}$ \\
\hline Maximum & $\mathrm{aw}_{\mathrm{J}}, \mathrm{cw}_{\mathrm{J}}, \mathrm{ew}, \mathrm{a} \Delta \mathrm{w}, \mathrm{c} \Delta \mathrm{w}, \mathrm{e} \Delta \mathrm{w}$ & 0.066 & $\begin{array}{c}0.265 \\
(0.071)\end{array}$ & $\begin{array}{l}-0.035 \\
(0.013)\end{array}$ \\
\hline \multirow[t]{3}{*}{ Preferred } & $\mathrm{w}_{\mathrm{J}} \mathrm{y}_{\mathrm{J}}, \Delta \mathrm{w}_{\mathrm{J}} \mathrm{y}_{\mathrm{J}}$ & 0.059 & $\begin{array}{c}0.177 \\
(0.068)\end{array}$ & $\begin{array}{c}0.102 \\
(0.062)\end{array}$ \\
\hline & ace, $a^{2} c, a^{2} e, a c^{2}, c^{2} e, a e^{2}, c^{2}$ & 0.041 & $\begin{array}{l}0.175 \\
(0.069)\end{array}$ & $\begin{array}{c}0.104 \\
(0.062)\end{array}$ \\
\hline & $\begin{array}{r}\text { aY80, cY80, e80, aY90, cY90, } \\
\text { e90, aY99, cY99, e99 }\end{array}$ & 0.028 & $\begin{array}{l}0.180 \\
(0.079)\end{array}$ & $\begin{array}{c}0.076 \\
(0.065)\end{array}$ \\
\hline
\end{tabular}

Notes: The elasticities are evaluated for a linear budget set at the pooled sample mean, i.e. $a=43.812$, $c=0.865, e=10.287, w=43.382, y=157,942$, and $h=1,475$. Standard errors are constructed using the bootstrap (1,000 replications) and are reported in parentheses. 
The preferred wage and income elasticity estimates are plotted at different net post-VAT hourly wage rates and unearned income levels in Figure 3 to illustrate the variation with the point of evaluation. Other explanatory variables are still kept at the pooled sample mean, but hours of work are no longer fixed - predicted values, given explanatory variables, are used. The net wage is varied between the $5^{\text {th }}$ last-segment and the $95^{\text {th }}$ first-segment percentiles of the pooled sample along the $\mathrm{x}$-axis, where a vertical line marks the actual-segment median. The unearned income is kept at the $5^{\text {th }}$ first-segment percentile in the dotted lines, at the actual-segment median in the solid lines, and at the $95^{\text {th }}$ first-segment percentile in the dashed lines. Thick lines are used for the wage elasticity, and thin lines are used for the income elasticity.

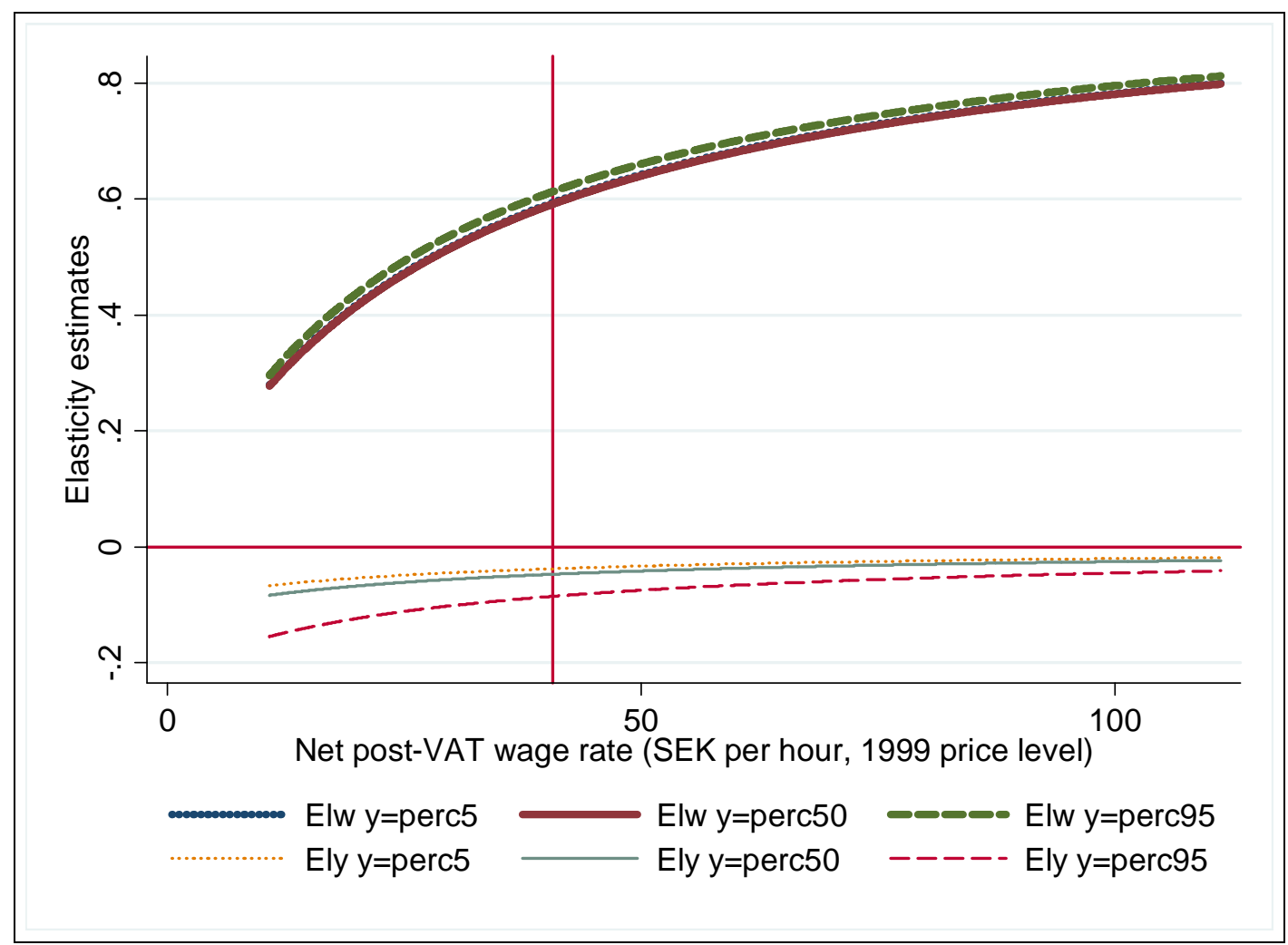

Figure 3. Elasticity estimates for linear budget sets at different wage rates and income levels

We see that the wage elasticity is positive and tends to increase in the net wage, but it does not vary much with the unearned income. The variation is large - from 0.3 to 0.8 . The income elasticity is negative and increases in the net wage but decreases in the unearned income. It varies between -0.2 and 0.0 . 


\subsection{Tax Systems and the Rise in Female Labor Supply}

The linear budget set elasticities used in the previous subsection should be interpreted with caution for reasons discussed in section three. It was argued that the labor supply regression estimates are more useful for simulations. Mongrel labor supply functions given the tax systems in 1973, 1980, 1990, and 1999 are predicted and plotted at different gross pre-PRT hourly wage rates in Figure 4 to compare the tax systems in the sample period. Other explanatory variables are kept at the pooled sample mean. The gross wage rate is varied from the $5^{\text {th }}$ to the $95^{\text {th }}$ pooled sample percentiles along the $\mathrm{x}$-axis, where a vertical line marks the median. A dotted line is used for 1973, a dashed line for 1980, a solid line for 1990, and a thick line for 1999.

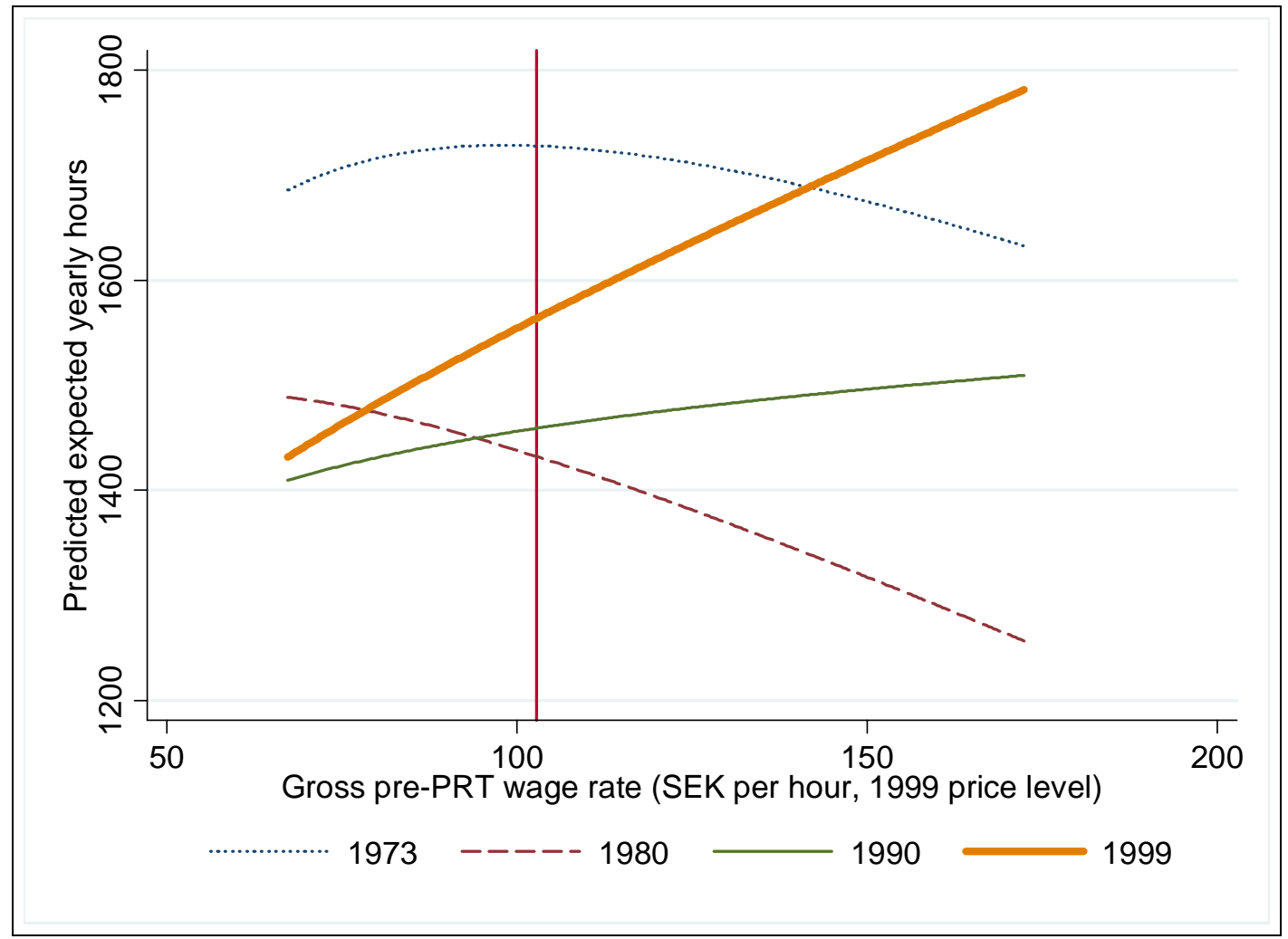

Figure 4. Predicted Mongrel labor supply functions for different years in the sample

We see that work incentives decreased until 1980 and increased thereafter. The incentives decreased in the gross wage in 1973 and 1980 but increased in the gross wage in 1990 and 1999. There is a close correspondence between the Mongrel labor supply functions and the marginal tax rates in Figure 2. Labor supply is high when tax rates are low, and high progressivity mainly discourages work for high income individuals. 
The actual variation in labor supply between the different years may also, depend on other factors, such as differences in gross incomes, demographics, time, or differences in the distribution of these variables. To sort out these effects, the Mongrel labor supply is predicted under different sets of explanatory variables in Table 4.

Table 4. Predicted expected yearly hours of work from 1973 to 1999

\begin{tabular}{l|cccc}
\hline Point of evaluation & 1973 & 1980 & 1990 & 1999 \\
\hline Pooled sample means & 1,727 & 1,426 & 1,461 & 1,571 \\
+ Yearly income variable means & 1,740 & 1,404 & 1,467 & 1,652 \\
+ Yearly control variable means & 1,234 & 1,445 & 1,711 & 1,903 \\
Distribution/Actual average & 1,145 & 1,384 & 1,666 & 1,812 \\
\hline
\end{tabular}

The years are varied horizontally, and the points of evaluation are varied vertically. The labor supply is first predicted at the pooled sample mean. The predicted variation across years reflects pure tax system differences. Next, the predictions are made at the yearly sample means for the gross pre-PRT income variables and then also for the control variables. The predicted variation additionally accounts for the average differences in gross incomes and then also in demographics and time factors between the years. Finally, the predictions are made for the actual sample individuals in the different years, given the different distribution of explanatory variables, and then averaged. The predicted variation then incorporates all systematic variation in the explanatory variables. These averages coincide with the actual averages.

At the pooled sample mean, labor supply falls dramatically until 1980, after which it increases. The rise is not as big as the fall. At the yearly means for the gross income variables, the rise after 1980 becomes larger. At the yearly means also for the control variables, the fall until 1980 becomes a rise. Additionally, the rise after 1980 is more than doubled. This produces a strong positive trend over time. Further accounting for the distribution does not change the trend.

The actual rise in labor supply until 1980 is therefore entirely due to demographics and time factors. Tax reforms can, however, at the pooled sample mean, explain 33.8 percent of the actual rise in hours of work after 1980, particularly the rise after 1990. They can, together with the evolution of gross income variables, account for 58.1 percent of the rise. The rest is mostly due to demographics and time factors. The overall picture is that tax systems had a large labor supply effect during this period, but the dramatic rise was mainly driven by changes in gross incomes, demographics, and time factors. 


\section{Simulating Hypothetical Reforms}

\subsection{Simulated Reforms}

This section simulates the labor supply, government revenues, and the income distribution of married women and, with some additional assumptions, households, under different tax systems using the preferred labor supply regression estimates from the previous section. ${ }^{6}$ The simulations primarily aim at gaining additional understanding of the details of the vast reforms between 1980 and 1999, which have previously been shown to have contributed to the rise in female labor supply. A secondary aim is to gain some insights about the tax system today.

The evaluation of a reform depends on the population affected by it and the tax system that was in place previously in the present nonparametric setting. The same reform could therefore have different effects at different points in time. To reach the goals of the simulations in the best way, I choose to perform hypothetical reforms on the 1999 subsample and tax system that was used in the estimation. The simulations reveal the counterfactual situations of what would have happened had we had another tax system in 1999, e.g., one that resembles the 1980 tax system, i.e., if the vast reforms after 1980 had not been implemented. The 1999 situation is also informative of today's situation since 1999 is not too long ago and since the largest rise in female labor supply had already taken place (not much room for further increases). The tax system also changed very little after 1999 until the earned income tax credit reform in 2007, and it provides a good proxy of the pre-2007-reform situation, which is useful for understanding the latest reform that is currently receiving much attention. Actual reform simulations often depart from pre-reform samples further back in time (e.g., Blomquist et al. 2001 and Flood et al. 2007).

The hypothetical reforms are quite simply and transparently designed and consist of marginal earned income tax increases in different brackets in the 1999 earned income tax system. The 1999 pre-reform tax system and three simulated reformed systems are shown in Table 5. The reforms increase tax rates in the lowest income brackets in $+10 \%$ low, the medium income brackets in $+10 \%$ medium, and the highest income brackets in $+10 \%$ high. The earned income tax rates are uniformly increased by ten percentage points. Roughly, the lowest

\footnotetext{
${ }^{6}$ I have also performed simulations using estimates from a number of other specifications as sensitivity tests. These results are not reported here, but are available upon request. First, additional regressors on top of those in the preferred specification have been added. Second, additional control variables have been included linearly in the preferred specification. These are socio-economic, metropolitan, and county dummies. Third, each of the cross-sections from 1973, 1980, and 1990 has been separately dropped. The results are insensitive to these tests.
} 
brackets reach up to around one third, the medium brackets are limited to one third and two thirds, and the highest brackets are beyond two thirds of the average gross post-PRT taxable earned income.

Table 5. Marginal earned income tax rates under the different simulated tax systems

\begin{tabular}{c|cccc}
\hline Bracket (lower limit) & 1999 & $+10 \%$ low & $+10 \%$ medium & $+10 \%$ high \\
\hline 0 & 0.000 & 0.100 & 0.000 & 0.000 \\
8,700 & 0.114 & 0.214 & 0.114 & 0.114 \\
12,340 & 0.363 & 0.463 & 0.363 & 0.363 \\
68,000 & 0.284 & 0.284 & 0.384 & 0.284 \\
105,200 & 0.363 & 0.363 & 0.463 & 0.363 \\
110,800 & 0.394 & 0.394 & 0.494 & 0.394 \\
135,000 & 0.406 & 0.406 & 0.406 & 0.506 \\
203,800 & 0.375 & 0.375 & 0.375 & 0.475 \\
219,300 & 0.575 & 0.575 & 0.575 & 0.675 \\
245,000 & 0.563 & 0.563 & 0.563 & 0.663 \\
299,832 & 0.515 & 0.515 & 0.515 & 0.615 \\
360,000 & 0.565 & 0.565 & 0.565 & 0.665 \\
\hline
\end{tabular}

Notes: Gross post-PRT earned income tax bracket limits are in SEK (1999 price level). Marginal tax rates that are changed in the reforms are reported in italics.

The tax increases in the medium and high income brackets together create a tax system that is comparable to the 1980 tax system and provide insights concerning the vast reforms between 1980 and 1999. The tax increase in the low income brackets is similar to the reverse of the introduction of the earned income tax credit program in 2007.

\subsection{Effects on Labor Supply}

The simulations of female labor supply are reported in Table 6. For each tax system, sample averages of the individual Mongrel labor supply predictions are reported, even for the prereform system to ensure comparability with the reformed systems. For the pre-reform system in 1999, these averages of the predictions are close to the actual averages. Percentage changes relative to the pre-reform system are reported in parentheses. The (hypothetical) reforms affect the wives' hours of work through different channels. The direct effects on the wives are accounted for in the Direct rows. The indirect cross effects on the wives due to the mechanical effects on the husbands' incomes, which constitute part of the wives' unearned incomes, are additionally accounted for in the +Indirect rows. There are additional indirect cross effects on the wives due to behavioral effects on the husbands. These are not accounted for. 
Table 6. Average predicted Mongrel female labor supply

\begin{tabular}{ll|cccc}
\hline Specification & Effects & 1999 & $+10 \%$ low & $+10 \%$ medium & $+10 \%$ high \\
\hline $\mathrm{E}(\mathrm{h})$ & Direct & 1,812 & 1,729 & 1,735 & 1,680 \\
& & & $(-4.590)$ & $(-4.280)$ & $(-7.292)$ \\
& + Indirect & & 1,732 & 1,738 & 1,687 \\
& & & $(-4.415)$ & $(-4.114)$ & $(-6.924)$ \\
\hline Pr(h>0) & Direct & 0.982 & 0.960 & 0.961 & 0.965 \\
& & & $(-2.219)$ & $(-2.167)$ & $(-1.760)$ \\
& +Indirect & & 0.960 & 0.960 & 0.968 \\
& & & $(-2.248)$ & $(-2.195)$ & $(-1.475)$ \\
\hline $\mathrm{E}(\mathrm{h} \mid \mathrm{h}>0)$ & Direct & 1,843 & 1,798 & 1,803 & 1,738 \\
& & & $(-2.429)$ & $(-2.158)$ & $(-5.685)$ \\
& +Indirect & & 1,802 & 1,807 & 1,740 \\
& & & $(-2.216)$ & $(-1.956)$ & $(-5.584)$ \\
\hline $\mathrm{E}(\mathrm{h})^{*} \mathrm{~W}_{\text {pre-PRT }}$ & Direct & 264,805 & 252,886 & 253,644 & 242,693 \\
& & & $(-4.501)$ & $(-4.215)$ & $(-8.350)$ \\
& +Indirect & & 253,340 & 254,077 & 243,707 \\
& & & $(-4.329)$ & $(-4.051)$ & $(-7.967)$ \\
\hline
\end{tabular}

Notes: Average predicted expected yearly hours, participation probabilities, expected yearly hours for working individuals, and expected yearly gross pre-PRT taxable earned incomes in SEK (1999 price level) are reported. Percentage changes relative to the pre-reform system are reported in parentheses. Direct holds the husbands' incomes constant, whereas +Indirect accounts for the mechanical effects on the husbands' incomes.

The reforms have negative behavioral effects on both the participation and hour margins. The (marginal) tax (rate) increases in the low and medium income brackets have similar effects and the contributions from the two margins are roughly equal (-4.590 and -4.280 percent). The tax increase in the high income brackets is more than two thirds worse with respect to average expected hours (-7.292 percent). The response on the participation margin is, however, smaller (-1.760 compared to -2.219 and -2.167 percent) and the response on the hour margin much larger ( -5.685 compared to -2.429 and -2.158 percent) than in the lower income brackets. The participation response to tax rates in the high income brackets is, however, surprisingly high, and not much lower than the responses to tax rates in the lower income brackets. This indicates that the participation decision depends on the whole budget set, including half-time and full-time options, and not just the first-segment characteristics. Predicted average gross pre-PRT taxable earned income responds similarly as the predicted average labor supply, with the most notable difference being that the tax increase in high income brackets becomes worse. This indicates that high-income individuals who receive a higher weight are disproportionally harmed by this reform.

The additional indirect effects are very small. This indicates that income effects, in the sense of altered unearned incomes keeping the tax system intact elsewhere, are small. The additional cross-effects on the wives due to behavioral effects on the husbands are also un- 
earned income effects of this kind and therefore likely to be small if the behavioral effects on the husbands' incomes are of the same order as the mechanical effects or smaller.

The effects on the wives can be translated into effects at the household level. The average labor supply percentage figures then need to be scaled by a factor of $0.461^{7}$, and the average expected gross pre-PRT taxable earned income percentage figures by a factor of $0.414^{8}$.

Average predicted Mongrel female labor supply functions at different gross pre-PRT wage rates are plotted in Figure 5 to assess how the effects are distributed. Relative to Figure 4, the covariation between the gross wage and other individual characteristics has been imposed using the observed joint distribution. A moving-average smoother has been applied, and the observations have then been averaged within small intervals to obtain smooth curves. The gross wage is varied from the $5^{\text {th }}$ to the $95^{\text {th }}$ percentiles, and a vertical line marks the median. A thick line is used for the pre-reform system, a dotted line for the tax increase in low income brackets, a dashed line for the tax increase in medium income brackets, and a solid line for the tax increase in high income brackets.

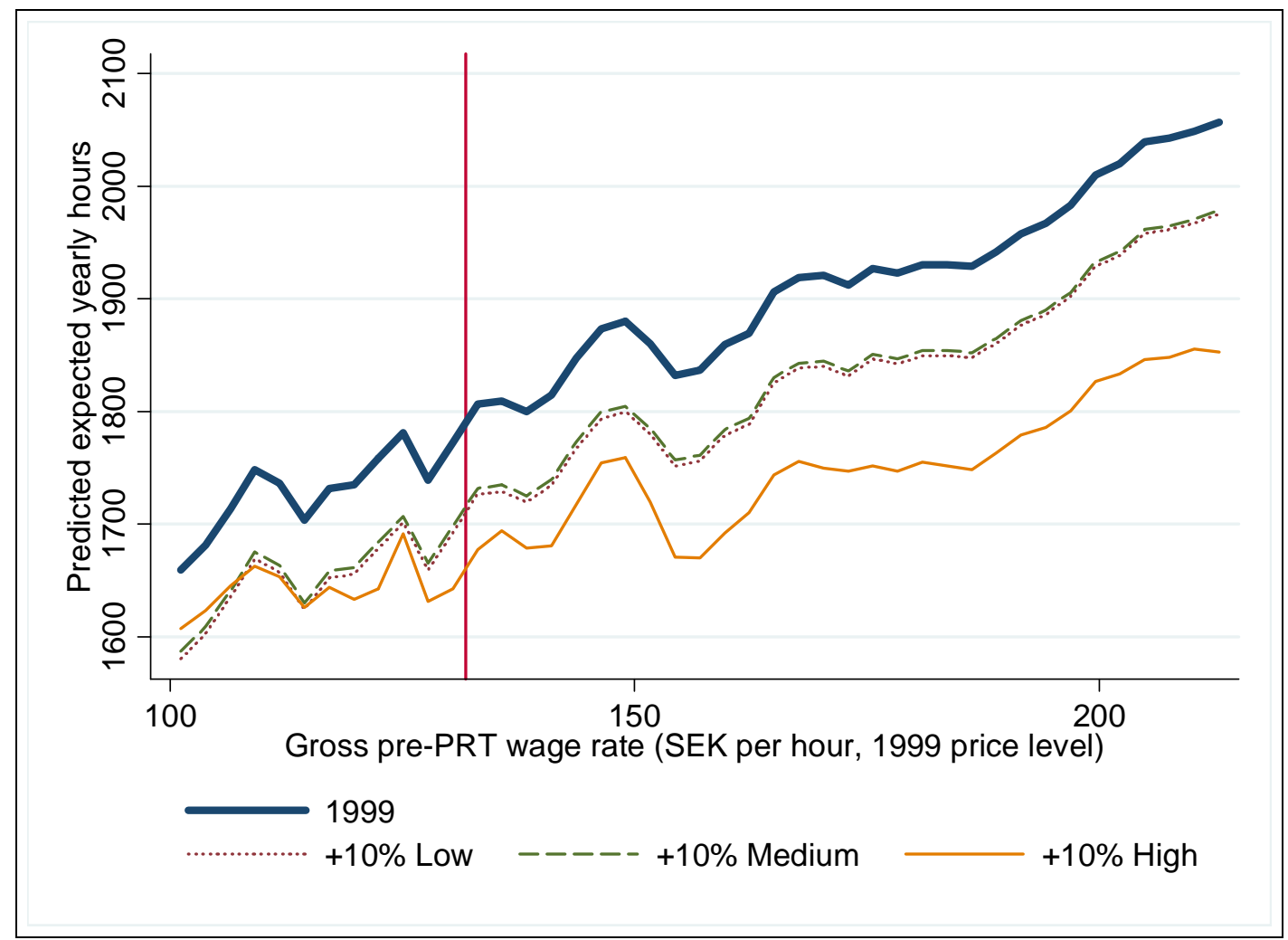

Figure 5. Average predicted Mongrel female labor supply functions across tax systems

\footnotetext{
${ }^{7}$ This is calculated using the fact that average yearly hours are 2,076 hours for a corresponding sample of married men.

${ }^{8}$ This is calculated using the fact that the average hourly gross pre-PRT wage is SEK 180.746 for a corresponding sample of married men.
} 
We see that the different reforms decrease expected hours of work at all gross wage rates. The decrease is fairly constant across gross wage rates for the tax increases in the low and medium income brackets, whereas it is larger at high wage rates for the tax increase in high income brackets. The tax increase in the high income brackets reduces expected hours of work more than the tax increases in the lower income brackets at all but the lowest gross wage rates.

\subsection{Effects on Incomes}

The individual Mongrel labor supply predictions can be used to predict individual incomes and government tax revenues, which can be averaged to obtain average effects. For the wives, the average predicted income is very close to the average actual income in the 1999 prereform system. For the husbands, actual individual gross pre-PRT taxable incomes in 1999 are instead used and held constant in the simulations.

The effects of the different tax systems on government revenue sources are reported in Table 7. To begin with, only the direct labor supply effects on the wives are included in the calculations. First, average earned income, capital income, payroll tax, and value added tax revenues per wife are reported. Then, transfer expenditures per household, which include child and housing allowances net value added tax, are reported. The sums of these posts are reported as net revenues from the wives. It is reasonable to regard transfer expenditures as pertaining to the wives here as the labor supply of the husbands is fixed, in which case any behavioral effects on transfer expenditures arise due to effects on the wives. To assess the importance of behavioral effects, net revenues assuming no labor supply effects from the reforms, i.e., with labor supply fixed to the pre-reform level, are then reported. The mechanical effects on the husbands are then accounted for. These have effects on the net revenues from the wives and from the husbands, which are both reported. Net revenues are finally reported at the household level with and without accounting for the direct labor supply effects on the wives. Percentage changes relative to the pre-reform system are reported within parentheses. 
Table 7. Average predicted government revenues

\begin{tabular}{l|cccc}
\hline Revenue source & 1999 & $+10 \%$ low & $+10 \%$ medium & $+10 \%$ high \\
\hline Earned income tax revenues Wife & 69,834 & 72,642 & 72,625 & 67,716 \\
& & $(+4.022)$ & $(+3.997)$ & $(-3.032)$ \\
Capital income tax revenues Wife & 1,819 & 1,819 & 1,819 & 1,819 \\
& & $( \pm 0.000)$ & $( \pm 0.000)$ & $( \pm 0.000)$ \\
Payroll tax revenues Wife & 65,793 & 62,832 & 63,020 & 60,299 \\
& & $(-4.501)$ & $(-4.215)$ & $(-8.350)$ \\
Value added tax revenues Wife & 17,026 & 15,621 & 15,695 & 15,196 \\
& & $(-8.250)$ & $(-7.817)$ & $(-10.746)$ \\
(-) Transfer expenditures & $-5,653$ & $-5,697$ & $-5,687$ & $-5,821$ \\
& & $(-0.778)$ & $(-0.602)$ & $(-2.959)$ \\
Net revenues Wife & 148,819 & 147,218 & 147,473 & 139,211 \\
& & $(-1.076)$ & $(-0.905)$ & $(-6.456)$ \\
Net revenues Wife & 148,819 & 154,572 & 154,372 & 154,795 \\
$\quad$ Fixed supply) & & $(+3.866)$ & $(+3.731)$ & $(+4.016)$ \\
\hline Net revenues Wife & 148,819 & 147,526 & 147,767 & 139,858 \\
$\quad$ (+Indirect) & & $(-0.869)$ & $(-0.707)$ & $(-6.022)$ \\
Net revenues Husband & 220,919 & 226,669 & 226,375 & 233,002 \\
& & $(+2.603)$ & $(+2.470)$ & $(+5.469)$ \\
Net revenues Household & 369,739 & 374,195 & 374,142 & 372,860 \\
& & $(+1.205)$ & $(+1.191)$ & $(+0.844)$ \\
Net revenues Household & 369,739 & 381,242 & 380,747 & 387,798 \\
$\quad$ (Fixed supply) & & $(+3.111)$ & $(+2.977)$ & $(+4.884)$ \\
\hline
\end{tabular}

Notes: Average predicted expected revenues in SEK (1999 price level) are reported. Percentage changes relative to the pre-reform system are reported within parentheses. +Indirect accounts for the mechanical effects on the husbands' incomes. Fixed supply holds labor supply fixed at the 1999 level.

The tax increases in the low and medium income brackets increase $(+4.022$ and +3.997 percent), whereas the tax increase in the high income brackets decreases (-3.032 percent) earned income tax revenues. Tax rates are therefore beyond the labor tax revenue maximum in the high income brackets. The negative behavioral effects decrease payroll tax and value added tax revenues and increase transfer expenditures. These losses more than counteract the earned income tax revenue gains, and net revenues ends up negative for tax increases in any income brackets $(-1.076,-0.905$, and -6.456 percent, respectively). The net revenue loss is five times larger in the high income brackets than in the lower income brackets. This indicates that tax rates are beyond the net tax revenue maximum for the wives. This can be compared to the net revenue gain from the reforms when not accounting for behavioral effects $(+3.866,+3.731$, and +4.016 percent). The mechanical losses from the wives are therefore wiped out by the gains from the behavioral effects.

The adjustment in revenues from the wives is small when accounting for the mechanical effects on the husbands' incomes. Those effects do, however, increase the revenues from the husbands. The percentage revenue gain is smaller in the low and medium income brackets and 
higher in the high income brackets $(+2.603$ and +2.470 compared to +5.469 percent $)$ in relation to the equivalent mechanical fixed supply increase for the wives $(+3.866$ and +3.731 compared to +4.016 percent). This is because the husbands' incomes are higher than the wives'. At the household level, all reforms increase net revenues $(+1.205,+1.191$, and +0.844 percent), indicating that tax rates are on the right side of the net revenue maximum.

Compared to the fixed labor supply case, we see, however, that the behavioral effects of the wives negate between $59.993(1-1.191 / 2.977)$ and $82.719(1-0.844 / 4.884)$ percent of the net revenue gains from the households. This is under the assumption that there are no behavioral effects on the husbands. This may be reasonable for the tax increases in the low and medium income brackets as those reforms primarily induce an income effect on the almost exclusively full-time working Swedish husbands with high incomes and as the income effects previously found for them are small (e.g., by Blomquist and Hansson-Brusewitz, 1990, with the Hausman approach, and by Blomquist and Newey, 2002, with a nonparametric approach). If this income effect is negative, there are even more net revenue gains from the husbands. ${ }^{9}$ However, such reasoning cannot be applied for the tax increase in the high income brackets. Blomquist and Newey (2002) find, e.g., that roughly 28 percent of the tax revenue change is counteracted by behavioral effects for Swedish men for tax increases in high income brackets. Accounting for this would almost exactly cancel out the remaining net revenue gain from the households ${ }^{10}$, indicating that tax rates in the high income brackets are right at the net revenue maximum for the households.

The tax increases and the labor supply decreases also decrease net incomes for the individuals. The average net post-VAT income of the wives without accounting for indirect effects decreases by $8.834,8.399$, and 10.856 percent for the tax increases in the low, the medium, and the high income brackets, respectively. Corresponding decreases at the household level, including the mechanical effects on the husbands, are 6.105, 5.801, and 9.399 percent, respectively. These losses are much larger than the eventual gains in net government revenues.

Gini coefficients among married women and households are reported in Table 8. This coefficient measures the area between the actual wage distribution and the 45-degree line and is a measure of income inequality. Many interesting income distribution effects are likely to

\footnotetext{
${ }^{9}$ Accounting for such effects would adjust the wives' unearned incomes and produce additional cross-effects. Such effects are, as already discussed, relatively small.

${ }^{10}$ A complete analysis would of course also require that we simultaneously account for cross-effects on the husbands due to mechanical and behavioral effects on the wives. If income effects on the husbands are small, as shown by the previous studies, these cross-effects are likely small for the husbands as well.
} 
take place among low income groups. Many of those groups are, however, excluded from the analysis here. The effects on married women and households alone may therefore not be of great interest in themselves, and the results should not be taken to reflect any general picture of the income distribution.

To begin with, the coefficient is reported for the wives' net post-VAT incomes in Wife, first with direct labor supply effects, then with labor supply fixed to the pre-reform level, and finally with direct and indirect labor supply effects. The coefficient is then reported for equivalent net post-VAT incomes in Equivalent. Equivalent net incomes are calculated by dividing household net incomes, including transfers, by the number of consumption units in each family, treating each adult as 0.96 and each child as 0.66 units as in Blomquist et al. (2001). Each household is then weighted by its size (the number of family members). The surplus or deficit that a reform creates is then assumed to be shared equally in per capita terms (through, e.g., less extensive public service) in the Surplus sharing specifications.

Table 8. Predicted Gini coefficients

\begin{tabular}{l|cccc}
\hline Unit and restriction & 1999 & $+10 \%$ low & $+10 \%$ medium & $+10 \%$ high \\
\hline Wife & 0.183 & 0.205 & 0.203 & 0.167 \\
$\quad$ Fixed supply & & 0.191 & 0.190 & 0.166 \\
$\quad$ Indirect & & 0.206 & 0.204 & 0.166 \\
Equivalent & 0.236 & 0.245 & 0.243 & 0.229 \\
$\quad$ Fixed supply & & 0.241 & 0.240 & 0.227 \\
Surplus sharing & & 0.239 & 0.238 & 0.226 \\
$\quad$ Surplus sharing + Fixed supply & & 0.229 & 0.228 & 0.208 \\
\hline
\end{tabular}

Notes: Gini coefficients are calculated for net post-VAT incomes for the wives in Wife and for equivalent incomes, which is net income per consumption unit where each adult is treated as 0.96 and each child as 0.66 units, weighting each household by its size in Equivalent. Fixed supply holds labor supply fixed at the 1999 level. Surplus sharing increases incomes with the average reform surplus per capita.

The tax increases in the low and medium income brackets increase the Gini coefficient and income inequality (from 0.183 to 0.205 and 0.203 , respectively) for the wives' net incomes, whereas the tax increase in the high income brackets decreases it (from 0.183 to 0.167). The change in income inequality in the low and medium income brackets is larger than when the labor supply is fixed (0.205 compared to 0.191 and 0.203 compared to 0.190$)$, indicating that behavioral effects worsen income inequality. The indirect effects are again small. The pattern is similar but less pronounced at the equivalent income level. Accounting for surplus and deficit sharing further weakens the reform effects on income inequality. Additionally, imposing a fixed labor supply decreases income inequality, making all three reforms income inequality decreasing (from 0.236 to $0.229,0.228$, and 0.208 , respectively), especially for the tax 
increase in the high income brackets. Increased taxation that is spent in a per capita fashion therefore enhances income equality when the labor supply is fixed. Behavioral effects may, however, counteract or reverse this effect, especially when tax rates are increased in the low or medium income brackets.

\subsection{Simulated Aggregate Elasticities}

Some of the results from the simulations of the hypothetical reforms can be summarized in terms of aggregate Mongrel elasticities, by shrinking the reforms to marginal changes while keeping the proportions of the different tax rate changes. Such elasticities, tied to the specific population and tax system in 1999 and the specific reforms, described by equations (13) to (18), are reported in Table 9. I report the uncompensated wage and income elasticities of labor supply at the different margins, as well as the net-of-tax elasticity of gross pre-PRT taxable income and the tax elasticity of net government tax revenues for the wives. For the wage elasticity, indirect effects due to the mechanical change in the husbands' incomes are excluded since they enter the wives' unearned incomes, which are by definition held fixed. The reforms do not involve any direct change in the unearned incomes of the wives that can be used to simulate the income elasticity. The indirect effects are instead used to simulate this elasticity. For the tax elasticity, indirect effects are not accounted for since I want to isolate pure effects on the wives here. However, the previous simulations show that the indirect effects are relatively small. To obtain a good summary measure at the household level would involve accounting not only for the indirect effects, but also the behavioral effects on the husbands.

Table 9. Aggregate Mongrel elasticities in 1999

\begin{tabular}{ll|ccc}
\hline Aggregate elasticity & Margin & $+10 \%$ Low & $+10 \%$ Medium & $+10 \%$ High \\
\hline $\mathrm{El}_{\mathrm{h} \mid \mathrm{w}}$ & $\mathrm{E}(\mathrm{h})$ & 0.885 & 0.868 & 1.356 \\
& $\operatorname{Pr}(\mathrm{h}>0)$ & 0.427 & 0.433 & 0.264 \\
& $\mathrm{E}(\mathrm{h} \mid \mathrm{h}>0)$ & 0.458 & 0.434 & 1.092 \\
$\mathrm{El}_{\mathrm{h} \mid \mathrm{y}}$ & $\mathrm{E}(\mathrm{h})$ & -0.046 & -0.046 & -0.046 \\
& $\operatorname{Pr}(\mathrm{h}>0)$ & 0.008 & 0.008 & 0.012 \\
& $\mathrm{E}(\mathrm{h} \mid \mathrm{h}>0)$ & -0.054 & -0.054 & -0.058 \\
\hline $\mathrm{El}_{\mathrm{Y} \mid 1-\tau}$ & & 0.897 & 0.884 & 1.605 \\
$\mathrm{El}_{\mathrm{T} \mid \tau}$ & & -0.282 & -0.261 & -1.471 \\
\hline
\end{tabular}

The elasticities are similar for marginal tax rate changes in the low and medium income brackets and larger in absolute terms in the high income brackets. In the low and medium 
income brackets, the wage elasticity is approximately 0.9 , with roughly equal contributions from the participation and hour margins. At the high income brackets, the wage elasticity is 1.4 , with the hour margin contributing four times more than the participation margin. This can be compared to the wage elasticity for linear budget sets, which ranges from 0.3 to 0.8 for most net wage rates (see Figure 3), where the contribution on the hour margin is roughly double that of the participation margin (see Table 2 and 3). The income elasticity is in the region of -0.05 , with the bulk made up of responses on the hour margin, and is close to the values obtained for linear budget sets.

The net-of-tax elasticity of gross pre-PRT taxable income is close to the wage elasticity of labor supply, which is of course because gross wages are treated as exogenous. The tax elasticity of net government revenues is negative for all reforms. This indicates that tax rates are beyond the revenue maximum for married women at all income levels. The elasticity is fairly small in the low and medium income brackets at approximately -0.3 , but large around -1.5 in the high income brackets. Decreasing marginal tax rates in the high income brackets such that the average tax rate decreases by one percent when there are no behavioral effects would therefore increase net government revenues by more than one percent when accounting for behavioral effects.

\section{Concluding Discussion}

This paper first extends the nonparametric approach to estimate labor supply developed by Blomquist and Newey (2002) to handle cases in which there are non-working individuals. In addition to estimation of expected hours of work, the extension enables estimation of the participation probability within the same framework, which makes it possible to sort out responses on the participation and hour margins. The method is then used to estimate the labor supply of married or cohabiting women in Sweden from 1973 to 1999. Despite the many limitations of using elasticities for linear budget sets as summary measures, I first construct such entities at the pooled sample mean and find a statistically significant uncompensated wage elasticity of 0.7 and a small income elasticity. The wage elasticity varies between 0.3 and 0.8 depending on the point of evaluation.

The wage elasticity that I find is large in comparison to the elasticity in the region of 0.1 for married Swedish men that Blomquist and Newey (2002) find, even when accounting for their higher average hours of work. It is, however, similar to what Kumar (2006) finds for 
married American women. The income elasticity was insignificant for Swedish men and in the region of -0.5 for American women in those studies. Further, the elasticities are very close to the wage elasticity of $0.35-0.70$ and the small income elasticity for married Swedish women that Blomquist and Hansson-Brusewitz (1990) find using the Hausman approach on similar data.

I also construct simulation-based aggregate elasticity measures specific to each tax system and population and to the kind of reform of interest. Such measures are more informative and policy relevant and are not associated with the interpretation and extrapolation problems plaguing linear budget set elasticities. For the 1999 tax system, which is similar to the present Swedish system, I find an aggregate uncompensated wage elasticity of 0.9 for marginal tax rate changes in low and medium income brackets and 1.4 in high income brackets. The hour margin contributes as much as the participation margin in low and medium income brackets, but four times more in high income brackets. I also find an aggregate income elasticity of -0.05 , with the bulk made up of responses on the hour margin.

Several things could be learned from the simulated aggregate elasticities. First, elasticities do not only vary with population and tax system, but also non-trivially with the kind of reform. Second, the hours of work decision for an individual may depend on other segments in the budget set than the segment in which they are located, which indicates the need to account for the whole budget set in the estimation. Third, elasticities that account for nonlinear budget sets may differ quite substantially from the commonly used linear budget set elasticities, which indicates the need to account for the whole budget set in constructing summary measures from the estimation. Finally, the low aggregate income elasticity found here suggests that cross effects of the husbands on the wives working through unearned incomes are small.

Another finding in the simulations is that marginal tax rates were beyond the revenue maximizing rates for married women in 1999. However, together with the results in Blomquist et al. (2001), the results here indicate that marginal tax rates are on the right side in low and medium income brackets and just at the revenue maximum in high income brackets at the household level. This is consistent with the finding in Holmlund and Söderström (2008) that top marginal tax rates were close to the revenue maximum in Sweden in the 1990s. They use a methodology that combines natural experiments and instrumental variables and also account for dynamics. The financial effects found here that the behavioral effects of the wives translate into are much larger than those of the husbands found in Blomquist et al. (2001), despite the wives' lower gross wages. 
Although there are large behavioral effects of taxation, the simulations also reveal that the dramatic evolution of the tax system can only explain a small share of the extreme rise in female labor supply during the sample period. Demographics and time factors, such as attitudes, technology, and macroeconomics, account for the main part of the rise.

The nonparametric method used here may be extended in several directions. One is to develop and estimate a household model. Another is to incorporate savings dynamics. For Sweden during the period of study, a few papers using the Hausman approach indicate that ignoring these aspects does not produce a large bias (see Blomquist and Hansson-Brusewitz, 1990; and Aronsson and Palme, 1998, for the household aspect and Flood and MaCurdy, 1992, for the dynamics aspect). Another potentially fruitful investigation would be to explore the possibilities of applying this nonparametric approach to taxable income. 


\section{Appendix}

\section{Appendix A}

The derivations of expected hours of work in Proposition 1 and the participation probability in Proposition 2 are similar to the derivation of expected hours when there is no censoring conducted in Blomquist and Newey (2002), albeit much messier. I start with the simpler derivation of the participation probability in Proposition 2 to illustrate the underlying procedure before turning to expected hours in Proposition 1. In the derivations, I only lay out the steps in detail for the three-segment case in order to focus on the essentials. The generalization to the $J$-segment case is straightforward.

For the three-segment case, the conditions for observed and desired hours in equations (3) and (4) become:

$$
\begin{gathered}
h=\left\{\begin{array}{l}
h^{*}(\boldsymbol{x}, \boldsymbol{z}, v, \varepsilon) \\
0
\end{array} \text { and } D(h>0)=\left\{\begin{array}{l}
1 \text { if } h^{*}>0 \Leftrightarrow \varepsilon>-\pi^{*}(\boldsymbol{x}, \boldsymbol{z}, v) \\
0 \text { if } h^{*} \leq 0 \Leftrightarrow \varepsilon \leq-\pi^{*}(\boldsymbol{x}, \boldsymbol{z}, v),
\end{array}\right.\right. \\
\pi^{*}(\boldsymbol{x}, \boldsymbol{z}, v)= \begin{cases}\pi_{1}(v) & \text { if }-\infty \leq v \leq \pi_{1}^{-1}\left(l_{1}\right) \\
l_{1} & \text { if } \pi_{1}^{-1}\left(l_{1}\right) \leq v \leq \pi_{2}^{-1}\left(l_{1}\right) \\
\pi_{2}(v) & \text { if } \pi_{2}{ }^{-1}\left(l_{1}\right) \leq v \leq \pi_{2}{ }^{-1}\left(l_{2}\right) \\
l_{2} & \text { if } \pi_{2}^{-1}\left(l_{2}\right) \leq v \leq \pi_{3}{ }^{-1}\left(l_{2}\right) \\
\pi_{3}(v) & \text { if } \pi_{3}{ }^{-1}\left(l_{2}\right) \leq v \leq \infty,\end{cases}
\end{gathered}
$$

Equations (A1) and (A2) can be collapsed into:

$$
h=\left\{\begin{array}{l}
\pi_{1}(v)+\varepsilon \\
l_{1}+\varepsilon \\
\pi_{2}(v)+\varepsilon \\
l_{2}+\varepsilon \\
\pi_{3}(v)+\varepsilon \\
0
\end{array} \text { and } D(h)=\left\{\begin{array}{l}
1 \text { if S1 } \Leftrightarrow-\infty \leq v \leq \pi_{1}^{-1}\left(l_{1}\right) \cap \varepsilon>-\pi_{1}(v) \\
1 \text { if } \mathrm{K} 1 \Leftrightarrow \pi_{1}^{-1}\left(l_{1}\right) \leq v \leq \pi_{2}{ }^{-1}\left(l_{1}\right) \cap \varepsilon>-l_{1} \\
1 \text { if } \mathrm{S} 2 \Leftrightarrow \pi_{2}^{-1}\left(l_{1}\right) \leq v \leq \pi_{2}^{-1}\left(l_{2}\right) \cap \varepsilon>-\pi_{2}(v) \\
1 \text { if } \mathrm{K} 2 \Leftrightarrow \pi_{2}{ }^{-1}\left(l_{2}\right) \leq v \leq \pi_{3}{ }^{-1}\left(l_{2}\right) \cap \varepsilon>-l_{2} \\
1 \text { if S3 } \Leftrightarrow \pi_{3}{ }^{-1}\left(l_{2}\right) \leq v \leq \infty \cap \varepsilon>-\pi_{3}(v) \\
0 \text { otherwise, }
\end{array}\right.\right.
$$

where $S i$ and $K i$ denote the conditions where individuals desire to locate on segment or kink $i$ and have positive observed hours.

Proof of Proposition 2. The participation probability can be obtained by summing the probabilities that desired hours are on each of the segments and kinks at the same time as observed hours are positive according to equation (A3): 


$$
\begin{aligned}
& \operatorname{Pr}(h>0 \mid \boldsymbol{x}, \boldsymbol{z})=\iint_{h>0} f(v, \varepsilon) d \varepsilon d v=\iint_{\mathrm{S} 1} f(v, \varepsilon) d \varepsilon d v+\iint_{\mathrm{K} 1} f(v, \varepsilon) d \varepsilon d v \\
& +\iint_{\mathrm{S} 2} f(v, \varepsilon) d \varepsilon d v+\iint_{\mathrm{K} 2} f(v, \varepsilon) d \varepsilon d v+\iint_{\mathrm{S} 3} f(v, \varepsilon) d \varepsilon d v \\
& =\int_{-\infty}^{\pi_{1}^{-1}\left(l_{1}\right)} \int_{-\pi_{1}(v)}^{\infty} f(v, \varepsilon) d \varepsilon d v+\int_{\pi_{1}^{-1}\left(l_{1}\right)}^{\pi_{-1}{ }^{-1}\left(l_{1}\right)} \int_{-l_{1}}^{\infty} f(v, \varepsilon) d \varepsilon d v \\
& +\int_{\pi_{2}{ }^{-1}\left(l_{1}\right)}^{\pi_{2}{ }^{-1}\left(l_{2}\right)} \int_{-\pi_{2}(v)}^{\infty} f(v, \varepsilon) d \varepsilon d v+\int_{\pi_{2}{ }^{-1}\left(l_{2}\right)}^{\pi_{3}{ }^{-1}\left(l_{2}\right)} \int_{-l_{2}}^{\infty} f(v, \varepsilon) d \varepsilon d v+\int_{\pi_{3}{ }^{-1}\left(l_{2}\right)}^{\infty} \int_{-\pi_{3}(v)}^{\infty} f(v, \varepsilon) d \varepsilon d v .
\end{aligned}
$$

The integrals are well-defined as the limits in the inner integrals only depend on the variable of integration in the outer integrals and as the limits in the outer integrals do not depend on the variables of integration.

Expanding some of the outer integrals in equation (A4) and summing over terms with similar outer integral limits gives:

$$
\begin{aligned}
& \operatorname{Pr}(h>0 \mid \boldsymbol{x}, \boldsymbol{z})=\int_{-\infty}^{\pi_{1}^{-1}\left(l_{1}\right)} \int_{-\pi_{1}(v)}^{\infty} f(v, \varepsilon) d \varepsilon d v \\
& +\int_{-\infty}^{\pi_{2}{ }^{-1}\left(l_{1}\right)} \int_{-l_{1}}^{\infty} f(v, \varepsilon) d \varepsilon d v-\int_{-\infty}^{\pi_{1}^{-1}\left(l_{1}\right)} \int_{-l_{1}}^{\infty} f(v, \varepsilon) d \varepsilon d v \\
& +\int_{-\infty}^{\pi_{2}{ }^{-1}\left(l_{2}\right)} \int_{-\pi_{2}(v)}^{\infty} f(v, \varepsilon) d \varepsilon d v-\int_{-\infty}^{\pi_{2}{ }^{-1}\left(l_{1}\right)} \int_{-\pi_{2}(v)}^{\infty} f(v, \varepsilon) d \varepsilon d v \\
& +\int_{-\infty}^{\pi_{3}{ }^{-1}\left(l_{2}\right)} \int_{-l_{2}}^{\infty} f(v, \varepsilon) d \varepsilon d v-\int_{-\infty}^{\pi_{2}^{-1}\left(l_{2}\right)} \int_{-l_{2}}^{\infty} f(v, \varepsilon) d \varepsilon d v \\
& +\int_{-\infty}^{\infty} \int_{-\pi_{3}(v)}^{\infty} f(v, \varepsilon) d \varepsilon d v-\int_{-\infty}^{\pi_{3}{ }^{-1}\left(l_{2}\right)} \int_{-\pi_{3}(v)}^{\infty} f(v, \varepsilon) d \varepsilon d v \\
& =\int_{-\infty}^{\infty} \int_{-\pi_{3}(v)}^{\infty} f(v, \varepsilon) d \varepsilon d v \\
& +\sum_{j=1}^{2} \int_{-\infty}^{\pi_{j}{ }^{-1}\left(l_{j}\right)} \int_{-\pi_{j}(v)}^{\infty} f(v, \varepsilon) d \varepsilon d v-\sum_{j=1}^{2} \int_{-\infty}^{\pi_{j}^{-1}\left(l_{j}\right)} \int_{-l_{j}}^{\infty} f(v, \varepsilon) d \varepsilon d v \\
& -\sum_{j=1}^{2} \int_{-\infty}^{\pi_{j+1}{ }^{-1}\left(l_{j}\right)} \int_{-\pi_{j+1}(v)}^{\infty} f(v, \varepsilon) d \varepsilon d v+\sum_{j=1}^{2} \int_{-\infty}^{\pi_{j+1}{ }^{-1}\left(l_{j}\right)} \int_{-l_{j}}^{\infty} f(v, \varepsilon) d \varepsilon d v \\
& =\int_{-\infty}^{\infty} \int_{-\pi_{3}(v)}^{\infty} f(v, \varepsilon) d \varepsilon d v \\
& +\sum_{j=1}^{2}\left\{\int_{-\infty}^{\pi_{j}^{-1}\left(l_{j}\right)}\left[\int_{-\pi_{j}(v)}^{\infty} f(v, \varepsilon) d \varepsilon-\int_{-l_{j}}^{\infty} f(v, \varepsilon) d \varepsilon\right] d v\right. \\
& \left.-\int_{-\infty}^{\pi_{j+1}}\left(l_{l_{j}}\right)\left[\int_{-\pi_{j+1}(v)}^{\infty} f(v, \varepsilon) d \varepsilon-\int_{-l_{j}}^{\infty} f(v, \varepsilon) d \varepsilon\right] d v\right\}
\end{aligned}
$$

Merging the inner integrals inside the summation gives:

$$
\begin{aligned}
& \operatorname{Pr}(h>0 \mid \boldsymbol{x}, \boldsymbol{z})=\int_{-\infty}^{\infty} \int_{-\pi_{3}(v)}^{\infty} f(v, \varepsilon) d \varepsilon d v \\
& +\sum_{j=1}^{2}\left[\int_{-\infty}^{\pi_{j}^{-1}\left(l_{j}\right)} \int_{-\pi_{j}(v)}^{-l_{j}} f(v, \varepsilon) d \varepsilon d v-\int_{-\infty}^{\pi_{j+1}^{-1}\left(l_{j}\right)} \int_{-\pi_{j+1}(v)}^{-l_{j}} f(v, \varepsilon) d \varepsilon d v\right] .
\end{aligned}
$$


From a dimensionality point of view, the derivation reduces a high-dimensional function to additive separable low-dimensional functions according to:

$$
\begin{aligned}
& \operatorname{Pr}(h>0 \mid \boldsymbol{x}, \boldsymbol{z})=s(\boldsymbol{x}, \boldsymbol{z})=s\left(w_{1}, w_{2}, w_{3}, y_{1}, y_{2}, y_{3}, l_{1}, l_{2}, \boldsymbol{z}\right) \\
& =\langle\text { eq.(A4) }\rangle=s_{1}\left(w_{1}, y_{1}, l_{1}, \boldsymbol{z}\right)+s_{2}\left(w_{1}, w_{2}, y_{1}, y_{2}, l_{1}, \boldsymbol{z}\right) \\
& +s_{3}\left(w_{2}, y_{2}, l_{1}, l_{2}, \boldsymbol{z}\right)+s_{4}\left(w_{2}, w_{3}, y_{2}, y_{3}, l_{2}, \boldsymbol{z}\right)+s_{5}\left(w_{3}, y_{3}, l_{2}, \boldsymbol{z}\right) \\
& =\langle\text { eq.(A5) and (A6) }\rangle \\
& =\bar{\pi}^{P}\left(w_{3}, y_{3}, \boldsymbol{z}\right)+\sum_{j=1}^{2}\left[\mu^{P}\left(w_{j}, y_{j}, l_{j}, \boldsymbol{z}\right)-\mu^{P}\left(w_{j+1}, y_{j+1}, l_{j}, \boldsymbol{z}\right)\right]
\end{aligned}
$$

The $J$-segment case is completely analogous, and equations (A6) and (A7) become:

$$
\begin{aligned}
& \operatorname{Pr}(h>0 \mid \boldsymbol{x}, \boldsymbol{z})=\bar{\pi}^{P}\left(w_{J}, y_{J}, \boldsymbol{z}\right)+\sum_{j=1}^{J-1}\left[\mu^{P}\left(w_{j}, y_{j}, l_{j}, \boldsymbol{z}\right)-\mu^{P}\left(w_{j+1}, y_{j+1}, l_{j}, \boldsymbol{z}\right)\right] \\
& \text { where } \bar{\pi}^{P}\left(w_{J}, y_{J}, \boldsymbol{z}\right)=\int_{-\infty}^{\infty} \int_{-\pi\left(w_{J}, y_{J}, z, v\right)}^{\infty} f(v, \varepsilon) d \varepsilon d v, \\
& \mu^{P}\left(w_{j}, y_{j}, l_{j}, \boldsymbol{z}\right)=\int_{-\infty}^{\pi^{-1}\left(w_{j}, y_{j}, l_{j}, z\right)} \int_{-\pi\left(w_{j}, y_{j}, z, v\right)}^{-l_{j}} f(v, \varepsilon) d \varepsilon d v,
\end{aligned}
$$

QED. Importantly for the estimation, the generalization from the three-segment to the $J$-segment case does not increase the dimensionality. Additional segments simply add terms to the difference sum without affecting its dimensionality.

Proof of Proposition 1. Expected hours of work can be obtained by summing integrals of observed hours times the density function, given that desired hours are on each of the segments and kinks and that observed hours are positive according to equation (A3), which is an application of the law of total expectation to the continuous case:

$$
\begin{aligned}
& E(h \mid \boldsymbol{x}, \boldsymbol{z})=\iint h f(v, \varepsilon) d \varepsilon d v=\iint_{h>0} h f(v, \varepsilon) d \varepsilon d v=\iint_{h>0} h^{*} f(v, \varepsilon) d \varepsilon d v \\
& =\iint_{\mathrm{S} 1}\left(h^{*} \mid \mathrm{S} 1\right) f(v, \varepsilon) d \varepsilon d v+\iint_{\mathrm{K} 1}\left(h^{*} \mid \mathrm{K} 1\right) f(v, \varepsilon) d \varepsilon d v \\
& +\iint_{\mathrm{S} 2}\left(h^{*} \mid \mathrm{S} 2\right) f(v, \varepsilon) d \varepsilon d v+\iint_{\mathrm{K} 2}\left(h^{*} \mid \mathrm{K} 2\right) f(v, \varepsilon) d \varepsilon d v+\iint_{\mathrm{S} 3}\left(h^{*} \mid \mathrm{S} 3\right) f(v, \varepsilon) d \varepsilon d v \\
& =\int_{-\infty}^{\pi_{1}{ }^{-1}\left(l_{1}\right)} \int_{-\pi_{1}(v)}^{\infty}\left[\pi_{1}(v)+\varepsilon\right] f(v, \varepsilon) d \varepsilon d v+\int_{\pi_{1}{ }^{-1}\left(l_{1}\right)}^{\pi^{-1}\left(l_{1}\right)} \int_{-l_{1}}^{\infty}\left[l_{1}+\varepsilon\right] f(v, \varepsilon) d \varepsilon d v \\
& \int_{\pi_{2}{ }^{-1}\left(l_{1}\right)}^{\pi^{-1}\left(l_{2}\right)} \int_{-\pi_{2}(v)}^{\infty}\left[\pi_{2}(v)+\varepsilon\right] f(v, \varepsilon) d \varepsilon d v+\int_{\pi_{2}{ }^{-1}\left(l_{2}\right)}^{\pi^{-1}\left(l_{2}\right)} \int_{-l_{2}}^{\infty}\left[l_{2}+\varepsilon\right] f(v, \varepsilon) d \varepsilon d v \\
& +\int_{\pi_{3}{ }^{-1}\left(l_{j-1}\right)}^{\infty} \int_{-\pi_{3}(v)}^{\infty}\left[\pi_{3}(v)+\varepsilon\right] f(v, \varepsilon) d \varepsilon d v .
\end{aligned}
$$


Equation (A9) has the same structure as equation (A4) but different integrands. Importantly, each integrand only depends on the same subset of variables as its integral limits. The integrands therefore do not add any dimensions to the integrals compared to the participation probability case.

Carrying out similar steps as in equation (A5) on equation (A9) and generalizing to $J$ segments gives:

$$
\begin{aligned}
& E(h \mid \boldsymbol{x}, \boldsymbol{z})=\bar{\pi}\left(w_{J}, y_{J}, \boldsymbol{z}\right)+\sum_{j=1}^{J-1}\left[\mu\left(w_{j}, y_{j}, l_{j}, \boldsymbol{z}\right)-\mu\left(w_{j+1}, y_{j+1}, l_{j}, \boldsymbol{z}\right)\right] \\
& \text { where } \bar{\pi}\left(w_{J}, y_{J}, \boldsymbol{z}\right)=\int_{-\infty}^{\infty} \int_{-\pi\left(w_{J}, y_{J}, z, v\right)}^{\infty}\left[\pi\left(w_{J}, y_{J}, \boldsymbol{z}, v\right)+\varepsilon\right] f(v, \varepsilon) d \varepsilon d v, \\
& \mu\left(w_{j}, y_{j}, l_{j}, \boldsymbol{z}\right)= \\
& \int_{-\infty}^{\pi^{-1}\left(w_{j}, y_{j}, l_{j}, z\right)}\left\{\int_{-\pi\left(w_{j}, y_{j}, z, v\right)}^{\infty}\left[\pi\left(w_{j}, y_{j}, \boldsymbol{z}, v\right)+\varepsilon\right] f(v, \varepsilon) d \varepsilon-\int_{-l_{j}}^{\infty}\left[l_{j}+\varepsilon\right] f(v, \varepsilon) d \varepsilon\right\} d v .
\end{aligned}
$$

QED. The derivation is completely identical to the derivation in equation (A7) for the participation probability with respect to the reduction in dimensionality.

\section{Appendix B}

Table A1. Sample selection

\begin{tabular}{l|ccccc}
\hline Observations & Pooled & 1973 & 1980 & 1990 & 1999 \\
\hline Original random sample & 26,578 & 6,593 & 6,813 & 6,710 & 6,462 \\
Responses & 21,675 & 5,616 & 5,613 & 5,306 & 5,140 \\
Women & 10,771 & 2,816 & 2,794 & 2,623 & 2,538 \\
Married or cohabiting & 7,095 & 1,882 & 1,798 & 1,723 & 1,692 \\
20-60 years old & 5,843 & 1,549 & 1,475 & 1,421 & 1,398 \\
No child $\leq 5$ years old & 4,253 & 1,075 & 1,083 & 1,041 & 1,054 \\
In the labor force & 3,610 & 959 & 902 & 960 & 789 \\
Not farmer or self-employed & 3,275 & 858 & 811 & 883 & 723 \\
No missing values & 2,882 & 825 & 746 & 698 & 613 \\
\hline
\end{tabular}




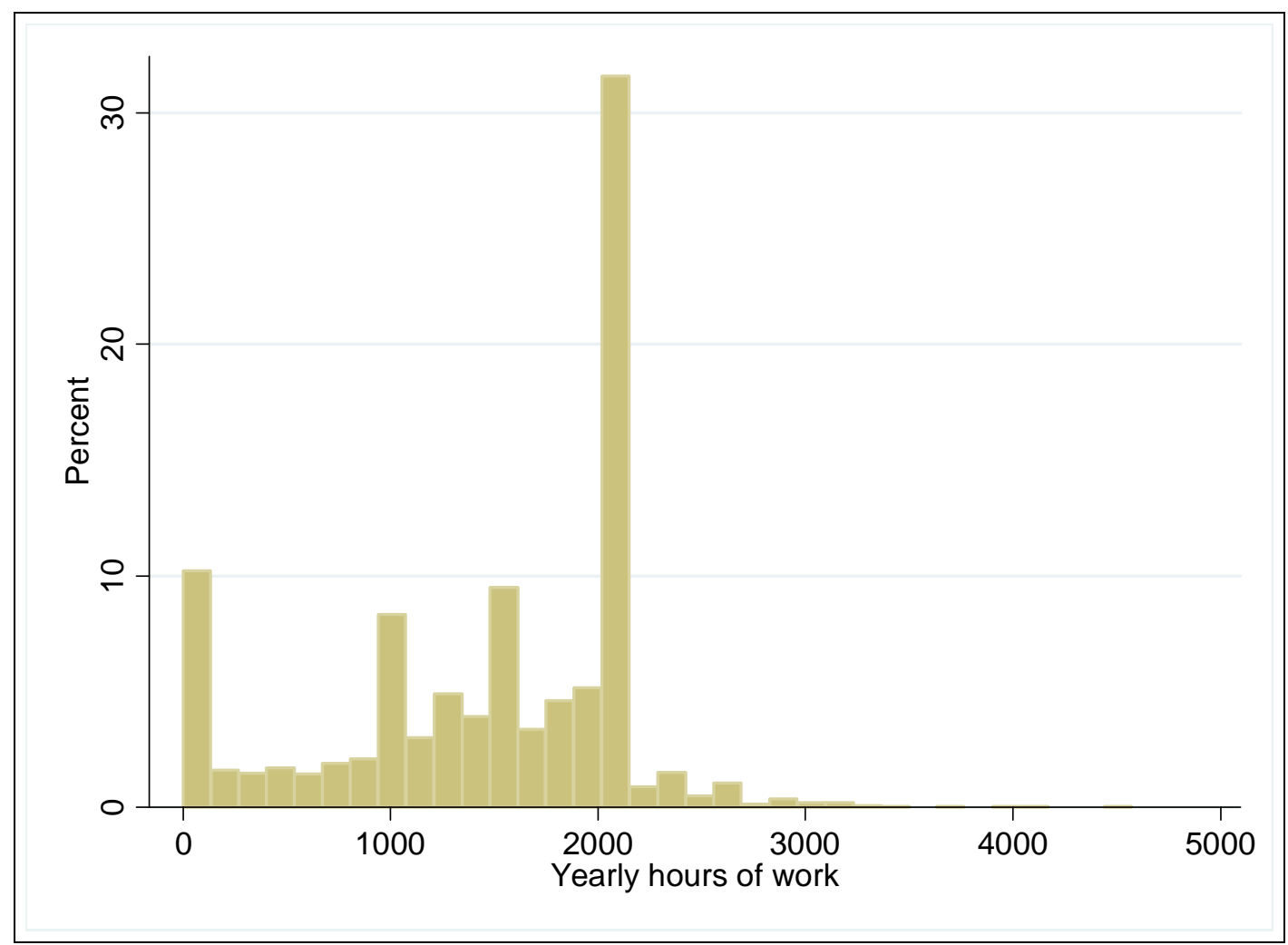

Figure A1. Distribution of hours of work

\section{Appendix C}

This appendix describes how the tax and transfer system in 1999 is implemented in the construction of the budget sets. The figures are expressed in SEK at the 1999 price level. Earned and capital incomes were separately taxed. The marginal earned income tax rates induced by different tax components are described in Table A2.

Table A2. Marginal earned income tax rates in 1999

\begin{tabular}{|c|c|c|c|c|c|}
\hline Bracket & Basic & Deduction & Pension fee & 99 EITC & Total \\
\hline 0 & 0.315 & -0.315 & & & 0 \\
\hline 8,700 & & 0 & $+0.685 * 0.0695$ & - All & 0 \\
\hline 12,340 & & & & 0 & 0.363 \\
\hline 68,000 & & $-0.25 * 0.315$ & & & 0.284 \\
\hline 105,200 & & 0 & & & 0.363 \\
\hline 110,800 & & $+0.10 * 0.315$ & & & 0.394 \\
\hline 135,000 & & & & +0.012 & 0.406 \\
\hline 203,800 & & 0 & & & 0.375 \\
\hline 219,300 & 0.515 & & $+0.485 * 0.0695$ & & 0.561 \\
\hline 245,000 & & & & 0 & 0.549 \\
\hline 299,832 & & & 0 & & 0.515 \\
\hline 360,000 & 0.565 & & & & 0.565 \\
\hline
\end{tabular}

Notes: Gross post-PRT taxable earned income tax bracket limits are in SEK (1999 price level). 
Basic includes an average local marginal tax of 0.315 and a national marginal tax of 0.20 for earned incomes above 219,300 and an additional 0.05 above 360,000. Local marginal tax rates varied little between local governments and were thickly clustered around the mean. Deduction pertains to basic deductions, which equaled the earned income for earned incomes up to $8,700,8,700$ up to $68,000,8,800+0.25 *$ (the earned income- 68,000 ) up to 105,200 , 18,000 up to $110,800,18,000-0.10^{*}$ (the earned income-105,200) up to 203,800 and 8,700 after 203,800. Pension fee was a general fee of 0.0695 on the earned income net basic tax for gross incomes less than 299,832. No fee was paid until the earned income reached 8,700. 99 EITC was an earned income tax credit of 1,320 that could be used against all tax payments. The credit decreased at a rate of 0.012 for earned incomes above 135,000. Total sums up the marginal tax rates in different income brackets from the different components.

Capital incomes were taxed at a rate of 0.3. Losses gave tax credits at the same rate for losses up to 100,000 and at the rate 0.21 for the part above 100,000. No refund was given if total tax payments turned negative. Capital losses therefore brought marginal tax rates to zero until the capital loss tax credit was fully exploited and shifted other tax credits upward.

The child allowance was the major allowance for families and was a general nonmeans-tested allowance. The allowance was 9,000 each year per child aged 15 years old or younger, plus an additional large family supplement of 2,400 for the third child, 7,200 for the fourth child, and 9,000 for the fifth child. 16- and 17-years-old children received 6,750 each year. I have access to and use actual payouts rather than numbers constructed from the children's ages.

The housing allowance was means-tested and is described in Table A3. Families with children or couples where both partners were under the age of 29 were eligible. A basic amount was first given. Another amount depended on family size. Of the subsidy-relevant monthly housing cost, 75 percent was subsidized within a certain interval, and 50 percent within another interval. These intervals are reported in $x \%$ of cost. The subsidy-relevant monthly housing cost was based on the rent or a constructed cost based on a number of factors, such as operation costs for house owners. Only the cost for the share of housing space below a maximum, reported in Max. space, entered the subsidy-relevant monthly cost. The full share was, however, used if the subsidy-relevant monthly cost was below the minimum reported in Min. cost. The allowance was then reduced by 20 percent of each of the spouses' incomes above 58,500 for families with children and 33.333 percent of household income above 58,000 for families without children as reported in Red. limit, and by 15 percent of household wealth above 100,000. 
Table A3. The housing allowance in 1999

\begin{tabular}{c|cccccc}
\hline Children & Basic & $75 \%$ of cost & $50 \%$ of cost & Max. space & Min. cost & Red. limit \\
\hline 0 & 0 & $1,800-2,600$ & $2,600-3,600$ & 60 & 0 & 58,000 \\
1 & 7,200 & $2,000-3,000$ & $3,000-5,300$ & 80 & 3,000 & 58,500 \\
2 & 10,800 & $2,000-3,300$ & $3,300-5,900$ & 100 & 3,300 & 58,500 \\
3 & 14,400 & $2,000-3,600$ & $3,600-6,600$ & 120 & 3,600 & 58,500 \\
4 & 14,400 & $2,000-3,600$ & $3,600-6,600$ & 140 & 3,900 & 58,500 \\
$\geq 5$ & 14,400 & $2,000-3,600$ & $3,600-6,600$ & 160 & 4,200 & 58,500 \\
\hline
\end{tabular}

Notes: Max. space is given in $\mathrm{m}^{2}$ and the other figures in SEK per year (1999 price level), except \% of cost and Min. cost, which refer to monthly costs. Families without children were only eligible if both spouses were under the age of 29 years old. Max. space refers to maximum space subsidized and Min. cost to minimum cost subsidized. Reduction rates with household income were 20 percent for families with children and 33.333 percent for families without children and start at Red. limit.

Payroll tax (PRT) amounted to 0.3306 of gross post-PRT income. Value added tax (VAT) was 0.25 of the pre-VAT price on most goods, 0.12 on some goods, such as food, and 0.06 on a few goods, such as books. I use the actual payroll tax rate, but a weighted value added tax rate calculated as aggregate value added tax revenues divided by aggregate private consumption in 1999 , which equaled 0.15551 of the pre-VAT price. 


\section{References}

Andrews, D. W. K., 1991, “Asymptotic Optimality of Generalized $\mathrm{C}_{\mathrm{L}}$, Cross-Validation, and Generalized Cross-Validation in Regressions with Heteroskedastic Errors”, Journal of Econometrics 47, 359-377.

Aronsson, T., and Palme, M., 1998, "A Decade of Tax and Benefit Reforms in Sweden: Effects on Labour Supply, Welfare and Inequality”, Economica 65, 39-67.

Auten, G., and Caroll, R, 1999, “The Effect of Income Taxes on Household Income”, Review of Economics and Statistics 81, 681-693.

Blomquist, N. S., 1983, “The Effect of Income Taxation on the Labor Supply of Married Men in Sweden", Journal of Public Economics 22, 169-197.

Blomquist, N. S., and Hansson-Brusewitz, U., 1990, "The Effect of Taxes on Male and Female Labor Supply in Sweden”, Journal of Human Resources 25, 317-357.

Blomquist, S., 1996, "Estimation Methods for Male Labor Supply Functions: How to Take Account of Nonlinear Taxes", Journal of Econometrics 70, 383-405.

Blomquist, S., Eklöf, M., and Newey, W., 2001, “Tax Reform Evaluation Using Non-Parametric Methods: Sweden 1980-1991”, Journal of Public Economics 79, 543-568.

Blomquist, S., and Newey, W., 2002, "Nonparametric Estimation with Nonlinear Budget Sets", Econometrica 70, 2455-2480.

Blomquist, S., and Selin, H., 2009, Hourly Wage Rate and Taxable Labor Income Responsiveness to Changes in Marginal Tax Rates, Working Paper 2009:1, Uppsala Center for Fiscal Studies, Department of Economics, Uppsala University.

Blundell, R., and MaCurdy, T., 1999, "Labor Supply: A Review of Alternative Approaches", in O. Ashenfelter and D. Card (eds.), Handbook of Labor Economics 3A, Elsevier North-Holland.

Burtless, G., and Hausman, J., 1978, "The Effect of Taxation on Labor Supply: Evaluating the Gary Negative Income Tax Experiment”, Journal of Political Economy 86, 1103-1130.

Chay, K., and Powell, J., 2001, "Semiparametric Censored Regression Models", Journal of Economic Perspectives 15, 29-42.

Das, M., Newey, W. K., and Vella, F., 2003, "Nonparametric Estimation of Sample Selection Models", Review of Economic Studies 70, 33-58.

Eissa, N., 1995, "Taxation and Labor Supply of Married Women: The Tax Reform Act of 1986 as a Natural Experiment,” NBER Working Paper 5023, National Bureau of Economic Research. 
Eklöf, M., and Sacklén, H., 1999, “The Hausman-MaCurdy Controversy: Why Do the Results Differ Across Studies", Journal of Human Resources 35, 204-220.

Feldstein, M., 1995, “The Effect of Marginal Tax Rates on Taxable Income: A Panel Study of the 1986 Tax Reform Act”, Journal of Political Economy 103, 551-572.

Flood, L., and MaCurdy, T., 1992, "Work Disincentive Effects of Taxes: An Empirical Analysis of Swedish Men”, Carnegie-Rochester Conference Series on Public Policy 37, 239-277.

Flood, L., Wahlberg, R., and Pylkkänen, E., 2007, "From Welfare to Work: Evaluating a Tax and Benefit Reform Targeted at Single Mothers in Sweden", Labour 21, 443-471.

Gelber, A. M., 2008, Taxation and Family Labor Supply, Manuscript, Harvard University.

Goolsbee, A., 1999, "Evidence on the High-Income Laffer Curve from Six Decades of Tax Reform", Brookings Papers on Economic Activity 2, 1-64.

Gruber, J., and Saez, E., 2002, "The Elasticity of Taxable Income: Evidence and Implications", Journal of Public Economics 84, 1-32.

Hansson, A., 2007, “Taxpayers Responsiveness to Tax Rate Changes and Implications for the Cost of Taxation", International Tax and Public Finance 14, 563-82.

Hausman, J., 1981, "Labor Supply”, in H. J. Aron and J. A. Pechman (eds.), How Taxes Affect Economic Behavior, Brookings Institution, 27-83.

Hausman, J., 1985, “The Econometrics of Nonlinear Budget Sets”, Econometrica 53, 12551282.

Heim, B. T., 2007, “The Incredible Shrinking Elasticities: Married Female Labor Supply, 1978-2002", Journal of Human Resources 42, 881-918.

Holmlund, B., and Söderström, M., 2007, "Estimating Income Responses to Tax Changes: A Dynamic Panel Data Approach”, Working Paper 2007:25, Department of Economics, Uppsala University.

Hoynes, H., 1996, "Welfare Transfers in Two-Parent Families: Labor Supply and Welfare Participation under the AFDC-UP Program”, Econometrica 64, 295-332.

Ichimura, H., 1993, "Semiparametric Least Squares (SLS) and Weighted SLS Estimation of Single-Index Models", Journal of Econometrics 58, 71-120.

Kumar, A., 2006, "Nonparametric Estimation of the Impact of Taxes on Female Labor Supply", Research Department Working Paper 0505, Federal Reserve Bank of Dallas.

Kumar, A., 2007, "Labor Supply, Deadweight Loss and Tax Reform Act of 1986: A Nonparametric Evaluation Using Panel Data”, Journal of Public Economics 92, 236- 253. 
Ljunge, M., and Ragan, K., 2006, Labor Supply and the Tax Reform of the Century?, Manuscript, University of Chicago.

MaCurdy, T., 1992, "Work Disincentive Effects of Taxes: A Reexamination of Some Evidence", American Economic Review 82, 243-249.

McDonald, J. F., and Mofitt, R., 1980, “The Uses of Tobit Analysis”, Review of Economics and Statistics 62, 318-321.

Mofitt, R. A., and Wilhelm, M. O., 2000, "Taxation and the Labor Supply Decisions of the Affluent", In J. Slemrod (ed.), Does Atlas Shrug? The Economic Consequences of Taxing the Rich, Harvard University Press.

Mroz, T. A., 1987, “The Sensitivity of An Empirical Model of Married Women's Hours of Work to Economic and Statistical Assumptions", Econometrica 55, 765-800.

OECD, 2008, Revenue Statistics 1965 - 2007, 2008 Edition, Organisation for Economic Cooperation and Development.

OECD, 2009, OECD StatExtracts, Organisation for Economic Co-operation and Development, http://stats.oecd.org/WBOS/index.aspx.

Pencavel, J., 1986, "Labor Supply of Men: A Survey”, O. Ashenfelter and R. Layard (eds.), Handbook of Labor Economics 1, Elsevier North-Holland.

Selén, J., 2004, Taxable Income Responses to Tax Changes: Panel Analyses of Swedish Reforms, Manuscript, Trade Union Institute for Economic Research.

Wooldridge, J. M., 2002, Econometric Analysis of Cross Section and Panel Data, MIT Press.

Van Soest, A., 1995, "Structural Models of Family Labor Supply: A Discrete Choice Approach”, Journal of Human Resources 30, 63-88.

Van Soest, A., Das, M., and Gong, X., 2002, “A Structural Labour Supply Model with Flexible Preferences", Journal of Econometrics 107, 345-374.

Wu, X., 2005, Labor Supply and Income Effects of the Earned Income Tax Credit and Welfare Programs, Manuscript, University of Guelph. 
WORKING PAPERS*

Editor: Nils Gottfries

2007:28 Che-Yuan Liang, Is There an Incumbency Advantage or a Cost of Ruling in Proportional Election Systems? 20pp.

2007:29 Stefan Eriksson and Jonas Lagerström, Detecting discrimination in the hiring process: Evidence from an Internet-based search channel. 31pp.

2007:30 Helge Berger and Pär Österholm, Does Money Growth Granger-Cause Inflation in the Euro Area? Evidence from Out-of-Sample Forecasts Using Bayesian VARs. 32pp.

2007:31 Ranjula Bali Swain and Maria Floro, Effect of Microfinance on Vulnerability, Poverty and Risk in Low Income Households. 35pp.

2008:1 Mikael Carlsson, Johan Lyhagen and Pär Österholm, Testing for Purchasing Power Parity in Cointegrated Panels. 20pp.

2008:2 Che-Yuan Liang, Collective Lobbying in Politics: Theory and Empirical Evidence from Sweden. 37pp.

2008:3 Spencer Dale, Athanasios Orphanides and Pär Österholm, Imperfect Central Bank Communication: Information versus Distraction. 33pp.

2008:4 Matz Dahlberg and Eva Mörk, Is there an election cycle in public employment? Separating time effects from election year effects. 29pp.

2008:5 Ranjula Bali Swain and Adel Varghese, Does Self Help Group Participation Lead to Asset Creation. 25pp.

2008:6 Niklas Bengtsson, Do Protestant Aid Organizations Aid Protestants Only? 28pp.

2008:7 Mikael Elinder, Henrik Jordahl and Panu Poutvaara, Selfish and Prospective Theory and Evidence of Pocketbook Voting. 31pp.

2008:8 Erik Glans, The effect of changes in the replacement rate on partial retirement in Sweden. 30pp.

2008:9 Erik Glans, Retirement patterns during the Swedish pension reform. 44pp.

2008:10 Stefan Eriksson and Jonas Lageström, The Labor Market Consequences of Gender Differences in Job Search. 16pp.

2008:11 Ranjula Bali Swain and Fan Yang Wallentin, Economic or Non-Economic Factors - What Empowers Women?. 34pp.

\footnotetext{
* A list of papers in this series from earlier years will be sent on request by the department.
} 
2008:12 Matz Dahlberg, Heléne Lundqvist and Eva Mörk, Intergovernmental Grants and Bureaucratic Power. 34pp.

2008:13 Matz Dahlberg, Kajsa Johansson and Eva Mörk, On mandatory activation of welfare receivers. $39 \mathrm{pp}$.

2008:14 Magnus Gustavsson, A Longitudinal Analysis of Within-Education-Group Earnings Inequality. 26pp.

2008:15 Henrique S. Basso, Delegation, Time Inconsistency and Sustainable Equilibrium. 24pp.

2008:16 Sören Blomquist and Håkan Selin, Hourly Wage Rate and Taxable Labor Income Responsiveness to Changes in Marginal Tax Rates. 31 pp.

2008:17 Jie Chen and Aiyong Zhu, The relationship between housing investment and economic growth in China : A panel analysis using quarterly provincial data. $26 \mathrm{pp}$.

2009:1 Per Engström, Patrik Hesselius and Bertil Holmlund, Vacancy Referrals, Job Search, and the Duration of Unemployment: A Randomized Experiment. $25 \mathrm{pp}$.

2009:2 Chuan-Zhong Li and Gunnar Isacsson, Valuing urban accessibility and air quality in Sweden: A regional welfare analysis. 24pp.

2009:3 Luca Micheletto, Optimal nonlinear redistributive taxation and public good provision in an economy with Veblen effects. $26 \mathrm{pp}$.

2009:4 Håkan Selin, The Rise in Female Employment and the Role of Tax Incentives. An Empirical Analysis of the Swedish Individual Tax Reform of $1971.38 \mathrm{pp}$.

2009:5 Lars M. Johansson and Jan Pettersson, Tied Aid, Trade-Facilitating Aid or Trade-Diverting Aid? 47pp.

2009:6 Håkan Selin, Marginal tax rates and tax-favoured pension savings of the selfemployed Evidence from Sweden. 32pp.

2009:7 Tobias Lindhe and Jan Södersten, Dividend taxation, share repurchases and the equity trap. $27 \mathrm{pp}$.

2009:8 Che-Yuan Liang, Nonparametric Structural Estimation of Labor Supply in the Presence of Censoring. 48pp.

See also working papers published by the Office of Labour Market Policy Evaluation http://www.ifau.se/ 